\title{
Adaptive Neural Network Fixed-Time Control Design for Bilateral Teleoperation with Time Delay
}

\author{
Shuang Zhang, Member, IEEE, Shuo Yuan, Student Member, IEEE, Xinbo Yu, Member, IEEE, Linghuan \\ Kong, Student Member, IEEE, Qing Li, Guang Li, Member, IEEE,
}

\begin{abstract}
In this paper, subject to time-varying delay and uncertainties in dynamics, we propose a novel adaptive fixed-time control strategy for a class of nonlinear bilateral teleoperation systems. First, an adaptive control scheme is applied to estimate the upper bound of delay, which can resolve the predicament that delay has significant impacts on stability of bilateral teleoperation systems. Then, radial basis function neural networks (RBFNNs) are utilized for estimating uncertainties in bilateral teleoperation systems including dynamics, operator and environmental models. Novel adaptation laws are introduced to address systems uncertainties in the fixed-time convergence settings. Next, a novel adaptive fixed-time neural network control scheme is proposed. Based on Lyapunov stability theory, the bilateral teleoperation systems are proved to be stable in fixed time. Finally, simulations and experiments are presented to verify the validity of the control algorithm.
\end{abstract}

Index Terms-Teleoperation, neural networks, fixed-time control, uncertainties, time delay.

\section{INTRODUCTION}

$\mathbf{T}$ ELEOPERATION systems can avoid direct touching between human operator and environment, and assist human operator to escape from harm caused by extreme environment [1], hence they have abstracted attentions from many scholars in recent years. A representative teleoperation system is composed of five component parts [2]: human operator, master robot, communication channel, slave robot and environment. The structure of bilateral teleoperation systems is shown in Fig. 1. The human operator operates master robot and drives it to move, thus master robot produces a series of track behaviors. These track behaviors will be transmitted to the slave robot controller through the forward communication channel. Then, the slave controller will give slave robot control signals to ensure that slave robot can move in accordance with the master robot. When the slave robot encounters environmental forces, position or force signals will be transmitted to master robot controller through the backward communication channel. The human operator can

This work was supported in part by the National Natural Science Foundation of China under Grant 61873297, and the Scientific and Technological Innovation Foundation of Shunde Graduate School of University of Science and Technology Beijing under Grant BK19BE015.

S. Zhang, S. Yuan and L. Kong are with School of Automation and Electrical Engineering, University of Science and Technology Beijing, Beijing 100083, China, and also with the Institute of Artificial Intelligence, University of Science and Technology Beijing, Beijing 100083, China. (Email: zhangshuang.ac@gmail.com)

$\mathrm{X}$. Yu is with Institute of Artificial Intelligence, University of Science and Technology Beijing, Beijing 100083, China.

Q. Li is with School of Automation and Electrical Engineering, University of Science and Technology Beijing, Beijing 100083, China.

G. Li is with School of Engineering and Materials Science, Queen Mary University of London, Mile End Road, London E1 4Ns, UK. make adjustments to action. There have been widely used for bilateral teleoperation systems in many fields, such as in space exploration [3], [4], medical devices [5], nuclear plants [6], deep ocean exploration [7], etc.

In actual situations, it is difficult to avoid time delay in data transmission. In some circumstances, introducing time delay into systems can improve control performance [8]-[10]. For example, in [8], an author introduced that delay can achieve vibration suppression in nonlinear systems with external excitation. Meanwhile, the time delay may have harmful impact on performance of systems [11]-[15]. In bilateral teleoperation systems, delay will affect the stability and operability [16]. To solve the issue, various control approaches have been raised. Passivity-based control theories [17]-[19] were widely used in bilateral teleoperation structure design for time delay compensation. A wave-variable control strategy [20] was applied to bilateral teleoperation systems to cope with instability caused by time delay. $\mathrm{P}+d$ and $\mathrm{PD}+d$ control strategies [21], [22] were designed in bilateral teleoperation systems with small external disturbances, such method can reduce the conservativeness of bilateral teleoperation compared to wave-variable control strategy. Robust control scheme [23] was proved to be an effective method to deal with time delay, in the case of small bound of time delay, such control method can obtain desired system performance. Zhang proposed proportional integral control scheme in [24] and used introduction of the upper bound of network-induced delays to ensure the systems exponentially stable, the conservativeness of systems was also reduced. Chopra et al. proposed an adaptive control strategy [25] for teleoperation systems with time delay. Many scholars have done a lot of research. For example, in [26], an adaptive fuzzy control was proposed for bilateral teleoperation systems with constant time delay. Wang et al. in [27] presented a new adaptive controller for bilateral teleoperation with time delay and backlash-like hysteresis. In this paper, an adaptive control scheme is applied to estimate the upper bound of the timevarying delay, which can ensure the stability of teleoperation systems and improve the performance of systems.

On the other hand, settling time is well recognized as one of the most criterion to evaluate the characteristics of bilateral teleoperation systems. Fast convergence time is pursued to obtain better performance and robustness. To achieve faster convergence performance, many approaches have been proposed [28]-[31]. For example, in [30], a switching strategy was investigated to obtain finite-time consensus for both continuous and discontinuous protocols on multiagent systems. In [31], a nonsingular terminal sliding mode (NTSM) control strategy was proposed in nonlinear systems to obtain a finitetime synchronization performance. However, the settling time 
of finite-time control method is dependent on initial values, which means different settling times are made by different system initial values. When information on initial states is unknown, the scope of finite-time control scheme will be limited. Fixed-time control method was first investigated by Polyakov in [32], the good point of fixed-time control scheme is that settling time is independent of initial states, which has important practical significance [33]. Based on this idea, fixedtime control techniques have been applied in various scenarios [34], [35].

In practice, it is difficult for us to acquire accurate dynamic models of teleoperation systems due to uncertainties in flexibilities and frictions of nonlinear robots [36]-[41]. Many approaches have been proposed to deal with uncertainties in nonlinear systems. Radial basis function neural networks (RBFNNs) method is considered as an effective way to deal with systems uncertainties, which requires relatively less information of the systems dynamics. It has been proved that RBFNNs method has the ability to approximate nonlinear functions with arbitrary accuracy [42], [43], thus it has been widely applied in uncertain nonlinear systems [44]-[49]. For example, an adaptive neural network controller [50] was put forward in multiple-input multiple-output (MIMO) nonlinear systems which require less system dynamic parameters. Yang et al. [51] proposed an neural networks control method to improve the performance of bilateral teleoperation systems. In this paper, RBFNNs method is used to estimate uncertainties in bilateral teleoperation systems including dynamics, operator and environmental models, different from off-line RBFNNs training relying on historical data. In our paper, we design online weight adaptation laws to minimize estimation errors, which can adjust RBFNNs weights according to timevarying environment or dynamics. Novel adaptation laws are introduced to address systems uncertainties in the fixed-time convergence settings. The stability of teleoperation systems is proved while high performance of tracking trajectory is obtained.

In this paper, with the aim of obtaining high tracking performances, we propose a novel adaptive neural network fixed-time control scheme for bilateral teleoperation systems considering time delay. The main contributions of this paper are summarized as follows:

First, we apply an adaptive control algorithm to estimate the upper bound of the time-varying delay to eliminate the impact of time-varying delay on stability of bilateral teleoperation systems.

Second, RBFNNs method is used to estimate uncertainties in bilateral teleoperation systems including dynamics, operator and environmental models. Different from off-line RBFNNs training relying on historical data, we design online weight adaptation laws to minimize estimation errors, which can adjust RBFNNs' weights according to time-varying environment or dynamics. Novel adaptation laws are introduced to address systems uncertainties in the fixed-time convergence settings. The stability of teleoperation systems is proved while high performance of tracking trajectory is obtained.

Third, we propose an adaptive neural network fixed-time control scheme in bilateral teleoperation nonlinear systems, with proposed control strategy, not only the stability of bi- lateral teleoperation nonlinear systems can be promised, but also error vectors of systems are able to converge to a small neighborhood around zero in fixed time.

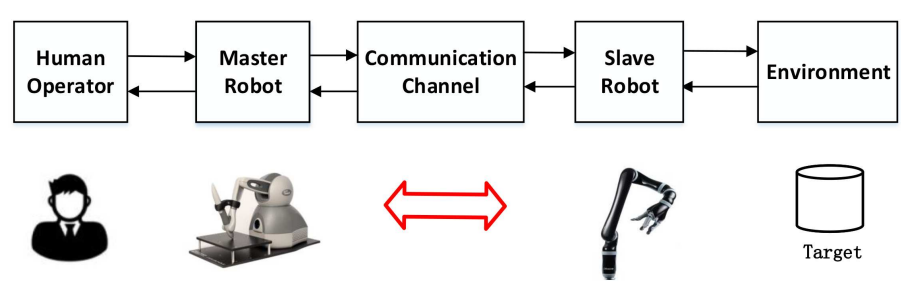

Fig. 1. Structure of teleoperation system.

\section{PReliminaries ANd PRoblem Formulation}

\section{A. Useful Technical Lemmas}

In order to facilitate future proofs, we summarize the following useful technical lemmas from literatures.

Lemma 1: [52] For any real numbers $m_{i}(i=1,2, \ldots, n)$ and $0 \leq a \leq 1$, We can get the following inequality

$$
\left(\sum_{i=1}^{n}\left|m_{i}\right|\right)^{a} \leq \sum_{i=1}^{n}\left|m_{i}\right|^{a}
$$

Lemma 2: [53] If $0<\alpha<1$, we have the following inequality

$$
\sum_{i=1}^{n}\left|m_{i}\right|^{1+\alpha} \geq\left(\sum_{i=1}^{n}\left|m_{i}\right|^{2}\right)^{\frac{1+\alpha}{2}}
$$

Lemma 3: If $m_{i} \geq 0$, we have

$$
\left(\sum_{i=1}^{n} m_{i}\right)^{2} \leq n \sum_{i=1}^{n} m_{i}^{2}
$$

which is well known as Cauchy-Schwarz inequality.

Lemma 4: [54] For $p, q \in \mathbb{R}$, we have

$$
p q \leq \frac{\varepsilon^{m}}{m}|p|^{m}+\frac{1}{n \varepsilon^{n}}|q|^{n}
$$

where $\varepsilon>0, m>1, n>1,(m-1)(n-1)=1$. This inequality is well known as Young's inequality.

Remark 1: When we choose $\varepsilon=1$ and $m=n=2$, we can simplify the expression of Lemma 4 as

$$
p q \leq \frac{1}{2}|p|^{2}+\frac{1}{2}|q|^{2}
$$

which are frequently used in the simplification of the paper.

Lemma 5: [55] For a class of nonlinear systems

$$
\dot{x}=f(x, t)
$$

where $x(0)=x_{0}, x \in \mathbb{R}^{+}$and $f: \mathbb{R}_{+} \times \mathbb{R}^{n} \rightarrow \mathbb{R}^{n}$. For $c>0, d>0, l>1,0<s<1$, if there is a positive definite and differentiable function $V(x)$, that satisfies

$$
\dot{V}(x) \leq-c V^{l}(x)-d V^{s}(x)
$$

then nonlinear systems are practically globally fixed-time stable. and the settling time $T$ can be expressed as

$$
T \leq T_{\max }=\frac{1}{c(l-1)}+\frac{1}{d(1-s)}
$$


Lemma 6: [56] For any vector $a \in \mathbb{R}^{n},\left(a^{T}\right)^{+}$is defined as the Moore-Penrose pseudo-inverse of $a^{T}$, the mathematical expression of $\left(a^{T}\right)^{+}$can be shown as

$$
\left(a^{T}\right)^{+}=\frac{a}{\|a\|^{2}}
$$

where $\|\bullet\|$ represents the standard Euclidean norm of $\bullet$. We can obtain the following property

$$
a^{T}\left(a^{T}\right)^{+}=\left\{\begin{array}{l}
0 \text { if } a=[0,0, \ldots, 0]^{T} \\
1 \text { otherwise }
\end{array}\right.
$$

\section{B. RBFNNS}

RBFNNs have strong generalization ability, many researches [57], [58] show that RBFNNs can approximate smooth nonlinear functions $f_{k}(x): \mathbb{R}^{q} \rightarrow \mathbb{R}$.

$$
f_{k}(x)=W_{k}^{T} \phi_{k}(x), k=1,2, \ldots, n
$$

where $x=\left[x_{1}, x_{2}, \ldots, x_{q}\right]^{T} \in \Omega_{Z} \subset \mathbb{R}^{q}$ stands for input parameter vector, $W_{i} \in \mathbb{R}^{l}$ represents the weight vector of the output layer. $l$ stands for the node number of neural network. $\phi_{k}(x)=\left[\varphi_{1}, \varphi_{2}, \ldots, \varphi_{l}\right]^{T} \in \mathbb{R}^{l}$ represents Gaussian function which can be described as

$$
\varphi_{h}(x)=\exp \left[\frac{-\left(x-c_{h}\right)^{T}\left(x-c_{h}\right)}{b_{h}{ }^{2}}\right], h=1,2, \ldots, l
$$

where $b_{h}$ is a positive scalar, representing the width of the gaussian function, $c_{m}=\left[c_{h 1}, c_{h 2}, \ldots, c_{h q}\right]^{T}$ stands for center vector.

If $l$ is selected sufficiently large, RBFNNs can approximate nonlinear functions with arbitrary precision over a compact set $\Omega \in \mathbb{R}^{q}$, and we have

$$
f_{k}(x)=W_{k}^{* T} \phi_{k}(x)+\epsilon_{k}(x)
$$

where $W_{k}^{*}$ stands for the ideal input parameter vector, $\epsilon_{k}(x) \in$ $\mathbb{R}$ is the approximation error of RBFNNs, $\left|\epsilon_{k}(x)\right| \leq \bar{\epsilon}_{k}$ and $\bar{\epsilon}_{k}$ is an unknown constant.

\section{Teleoperation Systems Dynamics}

Remark 2: In this paper, the subscript $i=m, s$ represents master and slave robot sides, $i^{\prime}=s, m$ are the opposite sides of $i . k=h, e$ represents human operator and environment side, which can simplify the expression of formula in the paper.

1) The dynamics model of master-slave teleoperation systems: Without considering disturbances and friction in teleoperation systems, the dynamic model of the teleoperation systems in the joint space can be described as

$$
\left\{\begin{array}{l}
M_{q m}\left(q_{m}\right) \ddot{q}_{m}+C_{q m}\left(q_{m}, \dot{q}_{m}\right) \dot{q}_{m}+G_{q m}\left(q_{m}\right) \\
=\tau_{m}+J_{m}^{T} f_{h} \\
M_{q s}\left(q_{s}\right) \ddot{q}_{s}+C_{q s}\left(q_{s}, \dot{q}_{s}\right) \dot{q}_{s}+G_{q s}\left(q_{s}\right) \\
=\tau_{s}-J_{s}^{T} f_{e}
\end{array}\right.
$$

where $q_{i} \in \mathbb{R}^{n}$ denotes joint position vector, $\dot{q}_{i} \in \mathbb{R}^{n}$ represents joint velocity vector and $\ddot{q}_{i} \in \mathbb{R}^{n}$ is joint acceleration vector. $M_{q i}\left(q_{i}\right) \in \mathbb{R}^{n \times n}$ denotes the inertia matrix, $C_{q i}\left(q_{i}, \dot{q}_{i}\right) \in \mathbb{R}^{n \times n}$ stands for the matrix of Coriolis and centripetal terms, $G_{q i}\left(q_{i}\right) \in \mathbb{R}^{n}$ represents the gravitational vectors, $\tau_{i} \in \mathbb{R}^{n}$ is the applied control input vector, $J_{i}$ denotes the Jacobian matrices are assumed to be bounded, $f_{h}$ is the force applied by operator to master robot and $f_{e}$ stands for the force exerted by environment to slave robot.

The dynamic model of the teleoperation systems has the following useful properties:

Property 1: [59] The matrix $M_{q i}\left(q_{i}\right)$ is a symmetric positive-definite matrix, and there exist positive constants $\lambda_{q i \min }$ and $\lambda_{q i \max }$ such that $0<\lambda_{q i \min } I \leq M_{q i}\left(q_{i}\right) \leq$ $\lambda_{q i \max } I$, where $I$ is identity matrix, $\lambda_{q i \min }$ and $\lambda_{q i \max }$ represent the minimum and maximum eigenvalues of $M_{q i}\left(q_{i}\right)$.

Property 2: [60] $\dot{M}_{q i}\left(q_{i}\right)-2 C_{q i}\left(q_{i}, \dot{q}_{i}\right)$ is skew-symmetric matrix and for any vector $z \in \mathbb{R}^{n}$, we have $z^{T}\left(\dot{M}_{q i}\left(q_{i}\right)-\right.$ $\left.2 C_{q i}\left(q_{i}, \dot{q}_{i}\right)\right) z=0$.

2) Communication channel: In bilateral teleoperation systems, the time-varing delays from forward and backward channel are represented by $\Delta T_{m}$ and $\Delta T_{s}$. We assume that $q_{m d}$ and $q_{s d}$ are desired trajectories of master and slave robot, we have

$$
\begin{aligned}
q_{m d} & =q_{s}\left(t-\Delta T_{s}\right) \\
q_{s d} & =q_{m}\left(t-\Delta T_{m}\right)
\end{aligned}
$$

Assumption 1: For the time-varying delay $\Delta T_{i}$, we assume that the following hypotheses

(1) $\Delta T_{i}$ is assumed to be bounded, which means

$$
0<\Delta T_{i}<T_{i \max }
$$

(2) The time-varying delay $\Delta T_{i}$ is differentiable and the derivative of $\Delta T_{i}$ is bounded. We assume that the derivative of $\Delta T_{i}$ can be represented by $\Delta \dot{T}_{i}$, we have

$$
\left|\Delta \dot{T}_{i}\right|<D_{i}
$$

where $D_{i}$ is unknown positive constant.

3) The dynamics of human operator and environment: The dynamics of human operator and environment may be inconsistent for different scenarios. References [61]-[63] introduce several dynamic models. In this paper, the dynamic models of human operator and environment are assumed to be linear time-invariant (LTI) descriptions, which are general dynamic models in the literature. The dynamics of LTI descriptions are as shown below

$$
\left\{\begin{array}{l}
f_{h}=f_{h}^{*}-M_{h} \ddot{x}_{m}-B_{h} \dot{x}_{m}-K_{h} x_{m} \\
f_{e}=f_{e}^{*}+M_{e} \ddot{x}_{s}+B_{e} \dot{x}_{s}+K_{e} x_{s}
\end{array}\right.
$$

where $M_{k}, B_{k}, K_{k}$ are positive-definite matrices corresponding to mass, damping and spring coefficient matrices of the operator and environment respectively. $x_{i} \in \mathbb{R}^{n}, \dot{x}_{i} \in \mathbb{R}^{n}$ and $\ddot{x}_{i} \in \mathbb{R}^{n}$ denote the position, velocity and acceleration vector in task space. $f_{h}^{*}$ and $f_{e}^{*}$ are nonhomogeneous terms, we assume that $f_{h}^{*}$ and $f_{e}^{*}$ are bounded, hence we have

$$
\left|f_{h}^{*}\right| \leq \eta_{h},\left|f_{e}^{*}\right| \leq \eta_{e}
$$

where $\eta_{h}$ and $\eta_{e}$ are known positive constants.

4) Transformation from task space to joint space: From the geometry of the robot structure we can know that there exist conversion relationships between the joint space and task space as

$$
x_{m}=H_{m}\left(q_{m}\right), x_{s}=H_{s}\left(q_{s}\right)
$$


where $H_{i}\left(q_{i}\right)$ is nonlinear transformation matrix which is used to describe coordinate transformation relationships between joint space and task space. Taking derivative of (20) we can obtain the velocity relationships as

$$
\dot{x}_{m}=J_{m}\left(q_{m}\right) \dot{q}_{m}, \dot{x}_{s}=J_{s}\left(q_{s}\right) \dot{q}_{s}
$$

where $J_{i}\left(q_{i}\right)$ is Jacobian matrix. Differentiating (21), we can get the acceleration relationships as

$$
\left\{\begin{array}{c}
\ddot{x}_{m}=\dot{J}_{m}\left(q_{m}\right) \dot{q}_{m}+J_{m}\left(q_{m}\right) \ddot{q}_{m} \\
\ddot{x}_{s}=\dot{J}_{s}\left(q_{s}\right) \dot{q}_{s}+J_{s}\left(q_{s}\right) \ddot{q}_{s}
\end{array}\right.
$$

Substituting (18), (20), (21) and (22) into (14), we can easily obtain

$$
\left\{\begin{array}{l}
M_{m}\left(q_{m}\right) \ddot{q}_{m}+C_{m}\left(q_{m}, \dot{q}_{m}\right) \dot{q}_{m}+G_{m}\left(q_{m}\right) \\
=\tau_{m}+\tau_{h} \\
M_{s}\left(q_{s}\right) \ddot{q}_{s}+C_{s}\left(q_{s}, \dot{q}_{s}\right) \dot{q}_{s}+G_{s}\left(q_{s}\right) \\
=\tau_{s}+\tau_{e}
\end{array}\right.
$$

where parameters in the formula have the following definitions

$$
\begin{aligned}
M_{i}\left(q_{i}\right) & =M_{q i}\left(q_{i}\right)+J_{i}^{T} M_{k} J_{i} \\
C_{i}\left(q_{i}, \dot{q}_{i}\right) & =C_{q i}\left(q_{i}, \dot{q}_{i}\right)+J_{i}^{T} M_{k} \dot{J}_{i}+J_{i}^{T} B_{k} J_{i} \\
G_{i}\left(q_{i}\right) & =G_{q i}+J_{i}^{T} K_{k} H_{i}\left(q_{i}\right) \\
\tau_{h} & =J_{m}^{T} f_{h}^{*} \\
\tau_{e} & =-J_{s}^{T} f_{e}^{*}
\end{aligned}
$$

According to the Property 1, Property 2 and matrix calculation, the combined model has the following properties.

Property 3: The inertia matrix $M_{i}\left(q_{i}\right)$ is positive-definite, and there exist positive constants $\lambda_{i \min }$ and $\lambda_{i \max }$ such that $0<\lambda_{i \min } I \leq M_{i}\left(q_{i}\right) \leq \lambda_{i \max } I$, where $I$ is identity matrix, $\lambda_{i \min }$ and $\lambda_{i \max }$ represent the minimum and maximum eigenvalues of $M_{i}\left(q_{i}\right)$.

Property 4: For any $x \in \mathbb{R}^{n}$, the $\dot{M}_{i}$ and $C_{i}\left(q_{i}, \dot{q}_{i}\right)$ satisfy the following formula

$$
x^{T}\left(\dot{M}_{i}\left(q_{i}\right)-2 C_{i}\left(q_{i}, \dot{q}_{i}\right)\right) x=-2 x^{T} J_{i}^{T} B_{k} J_{i} x \leq 0
$$

In practical applications, it is difficult to obtain accurate dynamic parameters in bilateral teleoperation systems. Therefore, uncertain parts are introduced into the description of teleoperation models. The dynamic parameters can be expressed as

$$
\begin{aligned}
M_{i}\left(q_{i}\right) & =M_{o i}\left(q_{i}\right)+\Delta M_{i}\left(q_{i}\right) \\
C_{i}\left(q_{i}, \dot{q}_{i}\right) & =C_{o i}\left(q_{i}, \dot{q}_{i}\right)+\Delta C_{i}\left(q_{i}, \dot{q}_{i}\right) \\
G_{i}\left(q_{i}\right) & =G_{o i}\left(q_{i}\right)+\Delta G_{i}\left(q_{i}\right)
\end{aligned}
$$

where $M_{o i}\left(q_{i}\right), C_{o i}\left(q_{i}, \dot{q}_{i}\right), G_{o i}\left(q_{i}\right)$ represent the nominal parts of dynamics and $\Delta M_{i}\left(q_{i}\right), \Delta C_{i}\left(q_{i}, \dot{q}_{i}\right), \Delta G_{i}\left(q_{i}\right)$ stand for the uncertainties of systems, then the dynamics of systems can be rewritten as

$$
\left\{\begin{array}{l}
M_{o m}\left(q_{m}\right) \ddot{q}_{m}+C_{o m}\left(q_{m}, \dot{q}_{m}\right) \dot{q}_{m}+G_{o m}\left(q_{m}\right) \\
=\tau_{m}+\tau_{h}-P_{m}\left(q_{m}, \dot{q}_{m}, \ddot{q}_{m}\right) \\
M_{o s}\left(q_{s}\right) \ddot{q}_{s}+C_{o s}\left(q_{s}, \dot{q}_{s}\right) \dot{q}_{s}+G_{o s}\left(q_{s}\right) \\
=\tau_{s}+\tau_{e}-P_{s}\left(q_{s}, \dot{q}_{s}, \ddot{q}_{s}\right)
\end{array}\right.
$$

where $P_{m}\left(q_{m}, \dot{q}_{m}, \ddot{q}_{m}\right)$ and $P_{s}\left(q_{s}, \dot{q}_{s}, \ddot{q}_{s}\right)$ are defined as

$$
\begin{aligned}
P_{m}\left(q_{m}, \dot{q}_{m}, \ddot{q}_{m}\right)= & \Delta M_{m}\left(q_{m}\right) \ddot{q}_{m}+\Delta C_{m}\left(q_{m}, \dot{q}_{m}\right) \dot{q}_{m} \\
& +\Delta G_{m}\left(q_{m}\right) \\
P_{s}\left(q_{s}, \dot{q}_{s}, \ddot{q}_{s}\right)= & \Delta M_{s}\left(q_{s}\right) \ddot{q}_{m}+\Delta C_{s}\left(q_{s}, \dot{q}_{s}\right) \dot{q}_{m} \\
& +\Delta G_{s}\left(q_{s}\right)
\end{aligned}
$$

\section{CONTRol Design AND ANALysis}

\section{A. Control Objectives}

We define the position synchronization error as

$$
z_{1 i}(t)=q_{i}(t)-q_{i d}(t)=q_{i}(t)-q_{i^{\prime}}\left(t-\Delta T_{i^{\prime}}\right)
$$

Differentiating (29), we have

$$
\dot{z}_{1 i}(t)=\dot{q}_{i}-\dot{q}_{i d}=\dot{q}_{i}(t)-\dot{q}_{i^{\prime}}\left(t-\Delta T_{i^{\prime}}\right)\left(1-\Delta \dot{T}_{i^{\prime}}\right)
$$

In practice application we can not get the exact value of $\Delta \dot{T}_{i}$, for that we define new velocity error as

$$
r_{i}(t)=\dot{q}_{i}(t)-\dot{q}_{i^{\prime}}\left(t-\Delta T_{i^{\prime}}\right)
$$

We introduce virtual control signal $\alpha_{i}(t)$, and define the second error variable as follows:

$$
z_{2 i}(t)=r_{i}(t)-\alpha_{i}(t)
$$

Combining (31) and (32) we have

$$
\dot{z}_{1 i}(t)=z_{2 i}(t)+\alpha_{i}(t)+\dot{q}_{i^{\prime}}\left(t-\Delta T_{i^{\prime}}\right) \Delta \dot{T}_{i^{\prime}}
$$

And we choose

$$
\alpha_{i}=-\left(\frac{1}{2}\right)^{\frac{3}{4}} k_{i 11}\left(z_{1 i}^{T}\right)^{+}\left(z_{1 i}^{T} z_{1 i}\right)^{\frac{3}{4}}-\left(\frac{1}{2}\right)^{2} k_{i 12} z_{1 i} z_{1 i}^{T} z_{1 i}
$$

where $k_{i 11}$ and $k_{i 12}$ are positive constants. With new variables defined in (32), the dynamics model of bilateral teleoperation systems can be transformed into

$$
\begin{aligned}
& M_{o i}\left(q_{i}\right) \dot{z}_{2 i}+C_{o i}\left(q_{i}, \dot{q}_{i}\right) z_{2 i}=\tau_{i}+\tau_{k}-G_{o i}\left(q_{i}\right) \\
& -M_{o i}\left(q_{i}\right) \dot{\alpha}_{i}-C_{o i}\left(q_{i}, \dot{q}_{i}\right) \alpha_{i}-Q_{i}
\end{aligned}
$$

where $Q_{i}$ has the following definition:

$$
\begin{aligned}
Q_{i}= & M_{o i} \ddot{q}_{i^{\prime}}\left(t-\Delta T_{i^{\prime}}\right)\left(1-\Delta \dot{T}_{i^{\prime}}\right)+C_{o i}\left(q_{i}, \dot{q}_{i}\right) \dot{q}_{i^{\prime}}\left(t-\Delta T_{i^{\prime}}\right) \\
& +P_{i}\left(q_{i}, \dot{q}_{i}, \ddot{q}_{i}\right)
\end{aligned}
$$

The main control objectives can be concluded as follows:

1) The bilateral teleoperation systems are stable.

2) The position synchronization error $z_{1 i}$ and velocity error $z_{2 i}$ can converge into neighbourhood around zero in fixed time.

3) The novel adaptive estimation laws are introduced to address systems uncertainties in the fixed-time convergence settings.

\section{B. Controller Design}

Consider dynamic uncertainties in bilateral teleoperation systems, we utilize neural networks to approximate the uncertainties as

$$
Q_{i}=W_{i}^{* T} \varphi_{i}\left(X_{i}\right)+\epsilon_{i}
$$

where $W_{i}^{* T}$ represents the ideal weight vector, $\varphi_{i}\left(X_{i}\right)$ is basis function. $\epsilon_{i} \in \mathbb{R}^{n}$ is inherent approximation error term and have upper bound that $\epsilon_{i} \leq \bar{\epsilon}_{i}$, the $\bar{\epsilon}_{i}$ is a positive constant. 
Definition 1: We have the following definition that

$$
\hat{W}_{i}^{T}=W_{i}^{* T}+\tilde{W}_{i}^{T}
$$

where $\hat{W}_{i}^{T}$ is the estimation of ideal weight $W_{i}^{* T}$ and $\tilde{W}_{i}^{T}$ is the neural network weight error.

The adaptive neural network fixed-time controllers are proposed as follows:

$$
\begin{aligned}
\tau_{i}= & -k_{i 21}\left(z_{2 i}^{T}\right)^{+}\left(z_{2 i}^{T} M_{o i} z_{2 i}\right)^{\frac{3}{4}}-k_{i 22}\left(z_{2 i}^{T}\right)^{+}\left(z_{2 i}^{T} M_{o i} z_{2 i}\right)^{2} \\
& +G_{o i}\left(q_{i}\right)+M_{o i}\left(q_{i}\right) \beta_{i}-\operatorname{sign}\left(z_{2 i}^{T}\right) \odot J_{i}^{T} \eta_{k}-\frac{1}{2} z_{2 i} \\
& +C_{o i}\left(q_{i}, \dot{q}_{i}\right) \alpha_{i}-\hat{D}_{i} N_{i} N_{i}^{T} z_{2 i}-z_{1 i}+\hat{W}_{i}^{T} \varphi\left(X_{i}\right)
\end{aligned}
$$

where $k_{i 21}$ and $k_{i 22}$ are positive constants, $\hat{W}_{i}^{T}$ stands for the estimated weight value of $W_{i}^{* T}$, From Assumption 1 we know $\left|\Delta \dot{T}_{i}\right| \leq D_{i}, \hat{D}_{i}$ is the estimated value of $D_{i}$, $N_{i}=\left(\left(z_{2 i}^{T}\right)^{+} z_{1 i}^{T}-M_{o i} f\left(z_{1 i}\right)\right) \dot{q}_{i^{\prime}}\left(t-\Delta T_{i^{\prime}}\right)$. The teleoperation systems with the new control algorithm is shown in Fig. 1.

Definition 2: For any vector $m, n \in \mathbb{R}^{n}$, the signal $\odot$ is defined as $m \odot n=\left[m_{1}, m_{2} \ldots, m_{n}\right] \odot\left[n_{1}, n_{2} \ldots, n_{n}\right]=$ $\left[m_{1} n_{1}, m_{2} n_{2} \ldots, m_{n} n_{n}\right]^{T}$

Remark 3: The signal $\operatorname{sign}(\bullet)$ returns a vector with the signs of the corresponding elements of the vector $(\bullet)$, combing Definition 1 we have

$$
z_{2 i}^{T}\left(-\operatorname{sign}\left(z_{2 i}^{T}\right) \odot J_{i}^{T} \eta_{k}+\tau_{k}\right) \leq 0
$$

Definition 3: We have the following definition that

$$
\hat{D}_{i}=D_{i}+\tilde{D}_{i}
$$

where $\hat{D}_{i}$ is estimation of $D_{i}$ and $\tilde{D}_{i}$ is estimation error of $D_{i}$.

Remark 4: In control design, we replace $\dot{\alpha}_{i}$ with $\beta_{i}$ to avoid introducing unknown delay change rate $\Delta \dot{T}_{i}$, which is unreasonable term appearing in controller. $\beta_{i}$ is defined as follows:

$$
\beta_{i}=f\left(z_{1 i}\right) r_{i}
$$

where $f\left(z_{1 i}\right)$ is derived from $\dot{\alpha}_{i}$, taking the derivative of (34), we have

$$
\begin{gathered}
\dot{\alpha}_{i}=f\left(z_{1 i}\right) \dot{z}_{1 i} \\
f\left(z_{1 i}\right)=k_{i 11}\left(-\left(\frac{1}{2}\right)^{\frac{3}{4}}\left(z_{1 i}^{T} z_{1 i}\right)^{-\frac{5}{4}}+\left(\frac{1}{2}\right)^{\frac{7}{4}} 3 z_{1 i} z_{1 i}^{T}\left(z_{1 i}^{T} z_{1 i}\right)^{-\frac{9}{4}}\right) \\
-\frac{1}{4} k_{i 12}\left(2 z_{1 i} z_{1 i}^{T}+z_{1 i}^{T} z_{1 i}\right)
\end{gathered}
$$

Remark 5: The values of $k_{i 11}, k_{i 12} k_{i 21}$ and $k_{i 22}$ have a great influence on the performance of our proposed controller. Increasing the values of them will yield a fast transient response and reduce the tracking errors in bilateral teleoperation systems. But large values of these parameters will make the control inputs large, which should be avoided if possible. We have tested different values of $k_{i 11}, k_{i 12} k_{i 21}$ and $k_{i 22}$ to find a good balance between these considerations.
The adaptive laws are given as follows:

$$
\begin{aligned}
\dot{\hat{D}}_{i}= & \lambda_{i}\left(z_{2 i}^{T} N_{i} N_{i}^{T} z_{2 i}-\mu_{i 1} \hat{D}_{i}-\mu_{i 2} \hat{D}_{i}^{3}\right) \\
\dot{\hat{W}}_{i j}= & -\Gamma_{i j}\left(\varphi_{i j}\left(X_{i}\right) z_{2 i, j}+\sigma_{i 1, j} \hat{W}_{i j}\right. \\
& \left.+\sigma_{i 2, j} \hat{W}_{i j} \hat{W}_{i j}^{T} \hat{W}_{i j}\right)
\end{aligned}
$$

where $j=1,2, \ldots, n . \lambda_{i}$ is a positive scalar, $\Gamma_{i j}$ is a constant gain matrix. $\mu_{i 1}, \mu_{i 2}, \sigma_{i 1, j}$ and $\sigma_{i 2, j}$ are small positive constants.

Remark 6: The novel adaptation laws are designed to guarantee errors of estimation upper bounds of time-varying delay $\tilde{D}_{i}$ and neural network weight errors $\tilde{W}_{i j}$ are able to converge to a small neighborhood around zero in fixed time.

\section{Stability analysis}

Consider a Lyapunov function candidate as

$$
V=V_{1}+V_{2}+V_{3}+V_{4}
$$

where $V_{1}, V_{2}, V_{3}$ and $V_{4}$ can be expressed as

$$
\begin{aligned}
V_{1} & =\sum_{i=m, s} \frac{1}{2} z_{1 i}^{T} z_{1 i} \\
V_{2} & =\sum_{i=m, s} \frac{1}{2} z_{2 i}^{T} M_{o i}\left(q_{i}\right) z_{2 i} \\
V_{3} & =\sum_{i=m, s} \frac{1}{2 \lambda_{i}} \tilde{D}_{i}^{2} \\
V_{4} & =\sum_{i=m, s} \frac{1}{2} \sum_{j=1}^{n} \tilde{W}_{i j}^{T} \Gamma_{i j}^{-1} \tilde{W}_{i j} \\
\dot{V} & =\dot{V}_{1}+\dot{V}_{2}+\dot{V}_{3}+\dot{V}_{4}
\end{aligned}
$$

Taking the derivative of $V_{1}$ we can get

$$
\dot{V}_{1}=\sum_{i=m, s} z_{1 i}^{T} \dot{z}_{1 i}
$$

Substituting (33) and (34) into (52), we have

$$
\begin{aligned}
\dot{V}_{1}= & \sum_{i=m, s}\left[z_{1 i}^{T} z_{2 i}+z_{1 i}^{T} \dot{q}_{i^{\prime}}\left(t-\Delta T_{i^{\prime}}\right) \Delta \dot{T}_{i^{\prime}}-k_{i 11}\left(\frac{1}{2} z_{1 i}^{T} z_{1 i}\right)^{\frac{3}{4}}\right. \\
& \left.-k_{i 12}\left(\frac{1}{2} z_{1 i}^{T} z_{1 i}\right)^{2}\right]
\end{aligned}
$$

Taking the derivative of $V_{2}$ respect to time, we can obtain

$$
\dot{V}_{2}=\sum_{i=m, s}\left[\frac{1}{2} z_{2 i}^{T} \dot{M}_{o i}\left(q_{i}\right) z_{2 i}+z_{2 i}^{T} M_{o i}\left(q_{i}\right) \dot{z}_{2 i}\right]
$$

From Property 4, we have $z_{2 i}^{T}\left(\frac{1}{2} \dot{M}_{o i}\left(q_{i}\right)-C_{o i}\left(q_{i}, \dot{q}_{i}\right)\right) z_{2 i} \leq$ 0 , and taking (39) into (54), we have

$$
\begin{aligned}
\dot{V}_{2}= & \sum_{i=m, s}\left[-k_{i 21}\left(\frac{1}{2} z_{2 i}^{T} M_{o i} z_{2 m}\right)^{\frac{3}{4}}-k_{i 22}\left(\frac{1}{2} z_{2 i}^{T} M_{o i} z_{2 i}\right)^{2}\right. \\
& +z_{2 i}^{T}\left(\tilde{W}_{i}^{T} \varphi_{i}\left(X_{i}\right)-\epsilon_{i}-z_{1 i}-\frac{1}{2} z_{2 i}-\hat{D}_{i} N_{i}^{T} N_{i} z_{2 i}\right. \\
& \left.\left.-\Delta \dot{T}_{i^{\prime}} M_{o i}\left(q_{i}\right) f\left(z_{1 i}\right) \dot{q}_{i^{\prime}}\left(t-\Delta T_{i^{\prime}}\right)\right)\right]
\end{aligned}
$$




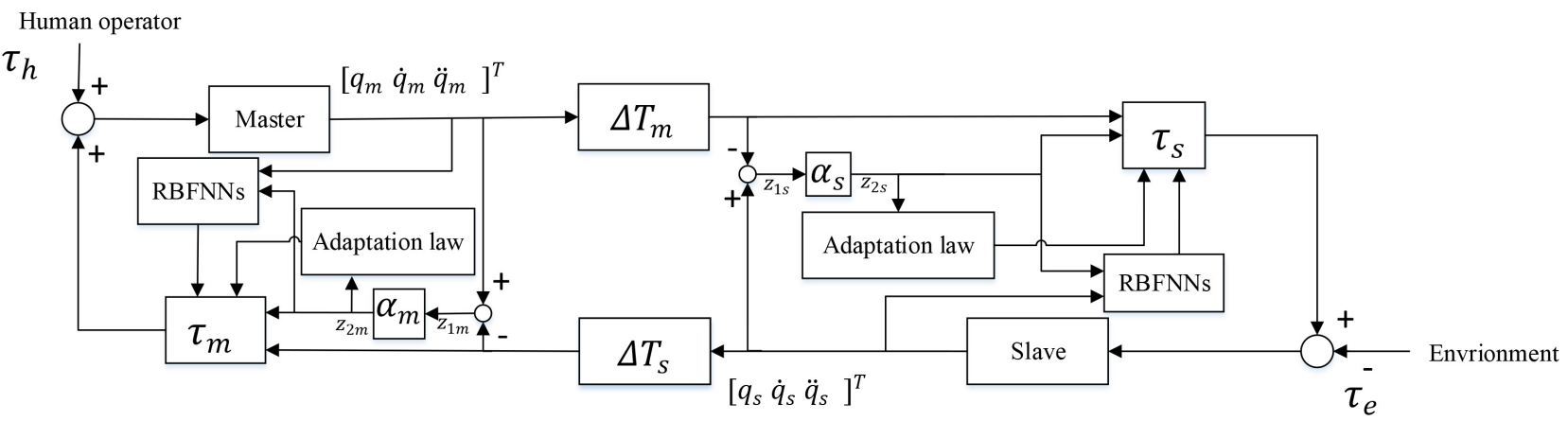

Fig. 2. Controller structure.

For $-z_{2 i}^{T} \epsilon\left(x_{i}\right)$, applying Lemma 4 , we can get

$$
-z_{2 i}^{T} \epsilon_{i} \leq \frac{1}{2} z_{2 i}^{T} z_{2 i}+\frac{1}{2}\left\|\bar{\epsilon}_{i}\right\|^{2}
$$

Substituting (56) into (55), hence $\dot{V}_{2}$ can be simplified as $\dot{V}_{2} \leq \sum_{i=m, s}\left[-k_{i 21}\left(\frac{1}{2} z_{2 i}^{T} M_{o i} z_{2 i}\right)^{\frac{3}{4}}-k_{i 22}\left(\frac{1}{2} z_{2 i}^{T} M_{o i} z_{2 i}\right)^{2}+\frac{1}{2}\left\|\bar{\epsilon}_{i}\right\|^{2}\right.$

$$
\begin{aligned}
& +z_{2 i}^{T}\left(\tilde{W}_{i}^{T} \varphi_{i}\left(X_{i}\right)-\Delta \dot{T}_{i^{\prime}} M_{o i}\left(q_{i}\right) f\left(z_{1 i}\right) \dot{q}_{i^{\prime}}\left(t-\Delta T_{i^{\prime}}\right)-z_{1 i}\right. \\
& \left.\left.-\hat{D}_{i} N_{i}^{T} N_{i} z_{2 i}\right)\right]
\end{aligned}
$$

Taking the derivative of $V_{3}$ respect to time, we can get

$$
\dot{V}_{3}=\sum_{i=m, s} \frac{1}{\lambda_{i}} \tilde{D}_{i} \dot{\hat{D}}_{i}
$$

Substituting (45) into (58), we have

$$
\dot{V}_{3}=\sum_{i=m, s} \tilde{D}_{i}\left(z_{2 i}^{T} N_{i} N_{i}^{T} z_{2 i}-\mu_{i 1} \hat{D}_{i}-\mu_{i 2} \hat{D}_{i}^{3}\right)
$$

For the term $-\mu_{i 1} \tilde{D}_{i} \hat{D}_{i}$, using Lemma 4 we have

$$
-\mu_{i 1} \tilde{D}_{i} \hat{D}_{i} \leq-\frac{\mu_{i 1}}{2} \tilde{D}_{i}^{2}+\frac{\mu_{i 1}}{2} D_{i}^{2}
$$

By method of completing the square, we can obtain

$$
\begin{aligned}
-\frac{\mu_{i 1}}{2} \tilde{D}_{i}^{2} \leq & -\frac{\mu_{i 1}}{4} \tilde{D}_{i}^{2}+\frac{\mu_{i 3}^{2}}{2 \sqrt{2} \mu_{i 1}}\left|\tilde{D}_{i}\right|-\mu_{i 3}\left(\frac{\tilde{D}_{i}^{2}}{2}\right)^{\frac{3}{4}} \\
& -\frac{1}{4}\left(\sqrt{\mu_{i 1}}\left|\tilde{D}_{i}\right|-2^{\frac{1}{4}} \frac{\mu_{i 3}}{\sqrt{\mu_{i 1}}} \sqrt{\left|\tilde{D}_{i}\right|}\right)^{2}
\end{aligned}
$$

where we choose $\mu_{i 3}>0$. It is just the term appeared in stability analysis, and have no concern with controller parameters.

For $\frac{\mu_{i 3}^{2}}{2 \sqrt{2} \mu_{i 1}}\left|\tilde{D}_{i}\right|$, after applying Lemma 4 we have

$$
\begin{aligned}
\frac{\mu_{i 3}^{2}}{2 \sqrt{2} \mu_{i 1}}\left|\tilde{D}_{i}\right| & =\frac{\sqrt{\mu_{i 1}}}{2}\left|\tilde{D}_{i}\right| \times \frac{\mu_{i 3}^{2}}{\sqrt{2} \mu_{i 1}^{\frac{3}{2}}} \\
& \leq \frac{\mu_{i 1}}{8}\left|\tilde{D}_{i}\right|^{2}+\frac{\mu_{i 3}^{4}}{4 \mu_{i 1}^{3}}
\end{aligned}
$$

Substituting (62) into (61), we have

$$
-\frac{\mu_{i 1}}{2} \tilde{D}_{i}^{2} \leq-\frac{\mu_{i 1}}{8}\left|\tilde{D}_{i}\right|^{2}+\frac{\mu_{i 3}^{4}}{4 \mu_{i 1}^{3}}-\mu_{i 3}\left(\frac{\tilde{D}_{i}^{2}}{2}\right)^{\frac{3}{4}}
$$

For the term $-\mu_{i 2} \tilde{D}_{i} \hat{D}_{i}^{3}$, we have

$$
\begin{aligned}
-\mu_{i 2} \tilde{D}_{i} \hat{D}_{i}^{3}= & -\mu_{i 2} \tilde{D}_{i}^{4}-3 \mu_{i 2} \tilde{D}_{i}^{3} D_{i}-3 \mu_{i 2} \tilde{D}_{i}^{2} D_{i}^{2} \\
& -\mu_{i 2} \tilde{D}_{i} D_{i}^{3}
\end{aligned}
$$

By Young's inequality in Lemma 4 , select $m=\frac{4}{3}$ and $n=4$ we have

$$
-3 \mu_{i 2} \tilde{D}_{i}^{3} D_{i} \leq 3 \mu_{i 2} \frac{3}{4} \varepsilon_{i}^{\frac{3}{4}}\left|\tilde{D}_{i}^{3}\right|^{\frac{4}{3}}+3 \mu_{i 2} \frac{1}{4 \varepsilon_{i}^{4}} D_{i}^{4}
$$

And

$$
-\mu_{i 2} \tilde{D}_{i} D_{i}^{3} \leq 3 \mu_{i 2} \tilde{D}_{i}^{2} D_{i}^{2}+\frac{\mu_{i 2}}{12} D_{i}^{4}
$$

We have

$-\mu_{i 2} \tilde{D}_{i} \hat{D}_{i}^{3} \leq-\mu_{i 2}\left(1-\frac{9 \varepsilon_{i}^{4}}{4}\right) \tilde{D}_{i}^{4}+\frac{\mu_{i 2}}{12} D_{i}^{4}+\frac{3 \mu_{i 2}}{4 \varepsilon_{i}^{4}} D_{i}^{4}$

Substituting (60), (63) and (67) into (59), we have

$$
\begin{aligned}
\dot{V}_{3} \leq & \sum_{i=m, s}\left[\tilde{D}_{i} z_{2 i}^{T} N_{i} N_{i}^{T} z_{2 i}-\mu_{i 3}\left(\frac{\tilde{D}_{i}^{2}}{2}\right)^{\frac{3}{4}}\right. \\
& \left.-\mu_{i 2}\left(1-\frac{9 \varepsilon_{i}^{4}}{4}\right) \tilde{D}_{i}^{4}+C_{i 3}\right]
\end{aligned}
$$

where $C_{i 3}=\frac{\mu_{i 1}}{2} D_{i}^{2}+\frac{\mu_{i 3}^{4}}{4 \mu_{i 1}^{3}}+\frac{\mu_{i 2}}{12} D_{i}^{4}+\frac{3 \mu_{i 2}}{4 \varepsilon_{i}^{4}} D_{i}^{4}$.

Taking the derivative of $V_{4}$ respect to time, we can get

$$
\dot{V}_{4}=\sum_{i=m, s}\left(\sum_{j=1}^{n} \tilde{W}_{i j}^{T} \Gamma_{i j}^{-1} \dot{\hat{W}}_{i j}\right)
$$

Employing the adaptation laws (45) into (69), we can obtain

$$
\begin{aligned}
\dot{V}_{4}= & \sum_{i=m, s}\left[\sum _ { j = 1 } ^ { n } \tilde { W } _ { i j } ^ { T } \Gamma _ { i j } ^ { - 1 } \left(-\Gamma_{i j}\left(\varphi_{i j}\left(X_{i}\right) z_{2 i, j}+\sigma_{i 1, j} \hat{W}_{i j}\right.\right.\right. \\
& \left.\left.\left.+\sigma_{i 2, j} \hat{W}_{i j} \hat{W}_{i j}^{T} \hat{W}_{i j}\right)\right)\right]
\end{aligned}
$$

After simplification, we can get

$$
\begin{aligned}
\dot{V}_{4}= & \sum_{i=m, s}\left[-z_{2 i}{ }^{T} \tilde{W}_{i}^{T} \varphi_{i}\left(X_{i}\right)-\sum_{j=1}^{n} \tilde{W}_{i j}^{T}\left(\sigma_{i 1, j} \hat{W}_{i j}\right.\right. \\
& \left.\left.+\sigma_{i 2, j} \hat{W}_{i j} \hat{W}_{i j}^{T} \hat{W}_{i j}\right)\right]
\end{aligned}
$$

According to Lemma 4, we have following inequalities:

$$
-\sigma_{i 1, j} \tilde{W}_{i j}^{T} \hat{W}_{i j} \leq-\frac{\sigma_{i 1, j}}{2}\left\|\tilde{W}_{i j}\right\|^{2}+\frac{\sigma_{i 1, j}}{2}\left\|W_{i j}^{*}\right\|^{2}
$$


Furthermore, selecting $\sigma_{i 3, j}>0$, we can get

$$
\begin{aligned}
-\frac{\sigma_{i 1, j}}{2}\left\|\tilde{W}_{i j}\right\|^{2}= & -\frac{\sigma_{i 1, j}}{4}\left\|\tilde{W}_{i j}\right\|^{2}+\frac{1}{2 \sqrt{2}} \frac{\sigma_{i 3, j}^{2}}{\sigma_{i 1, j}}\left\|\tilde{W}_{i i}\right\| \\
& -\sigma_{i 3, j}\left(\frac{1}{2}\left\|\tilde{W}_{i j}\right\|^{2}\right)^{\frac{3}{4}}-\frac{1}{4}\left(\sqrt{\sigma_{i 1, j}}\left\|\tilde{W}_{i j}\right\|\right. \\
& \left.-\frac{\sigma_{i 3, j}}{\sqrt{\sigma_{i 1, j}}} 2^{\frac{1}{4}} \sqrt{\left\|\tilde{W}_{i j}\right\|}\right)^{2}
\end{aligned}
$$

According to Lemma 4, we have:

$$
\frac{1}{2 \sqrt{2}} \frac{\sigma_{i 3, j}^{2}}{\sigma_{i 1, j}}\left\|\tilde{W}_{i j}\right\| \leq \frac{\sigma_{i 1, j}}{8}\left\|\tilde{W}_{i j}\right\|^{2}+\frac{\sigma_{i 3, j}^{4}}{4 \sigma_{i 1, j}^{3}}
$$

Substituting (74) into (73), we obtain

$$
-\frac{\sigma_{i 1, j}}{2}\left\|\tilde{W}_{i j}\right\|^{2} \leq \frac{\sigma_{i 3, j}^{4}}{4 \sigma_{i 1, j}^{3}}-\sigma_{i 3, j}\left(\frac{1}{2}\left\|\tilde{W}_{i j}\right\|^{2}\right)^{\frac{3}{4}}
$$

Expanding the following equalities, we can get

$$
\begin{aligned}
-\sigma_{i 2, j} & \tilde{W}_{i j}^{T} \hat{W}_{i j} \hat{W}_{i j}^{T} \hat{W}_{i j}=-3 \sigma_{i 2, j}\left\|\tilde{W}_{i j}\right\|^{2} \tilde{W}_{i j}^{T} W_{i j}^{*} \\
& -\sigma_{i 2, j}\left\|\tilde{W}_{i j}\right\|^{4}-\sigma_{i 2, j} \tilde{W}_{i j}^{T} W_{i j}^{*} \tilde{W}_{i j}^{T} W_{i j}^{*} \\
& -\sigma_{i 2, j} \tilde{W}_{i j}^{T} W_{i j}^{*} W_{i j}^{* T} \tilde{W}_{i j}-\sigma_{i 2, j}\left\|\tilde{W}_{i j}\right\|^{2}\left\|W_{i j}^{*}\right\|^{2} \\
& -\sigma_{i 2, j}\left\|W_{i j}^{*}\right\|^{2} \tilde{W}_{i j}^{T} W_{i j}^{*}
\end{aligned}
$$

For above equalities, we can obtain

$$
\begin{aligned}
-\sigma_{i 2, j} & \tilde{W}_{i j}^{T} \hat{W}_{i j} \hat{W}_{i j}^{T} \hat{W}_{i j} \leq-3 \sigma_{i 2, j}\left\|\tilde{W}_{i j}\right\|^{2} \tilde{W}_{i j}^{T} W_{i j}^{*} \\
& -\sigma_{i 2, j}\left\|\tilde{W}_{i j}\right\|^{4}-\sigma_{i 2, j}\left\|\tilde{W}_{i j}\right\|^{2}\left\|W_{i j}^{*}\right\|^{2} \\
& -\sigma_{i 2, j}\left\|W_{i j}^{*}\right\|^{2} \tilde{W}_{i j}^{T} W_{i j}^{*}
\end{aligned}
$$

Using Lemma 4, we can obtain

$$
\begin{array}{r}
-3 \sigma_{i 2, j}\left\|\tilde{W}_{i j}\right\|^{2} \tilde{W}_{i j}^{T} W_{i j}^{*} \leq 3 \sigma_{i 2, j}\left\|\tilde{W}_{i j}\right\|^{2}\left\|\tilde{W}_{i j}^{T}\right\|\left\|W_{i j}^{*}\right\| \\
\leq \frac{9}{4} \sigma_{i 2, j} \varepsilon_{i j}^{\frac{4}{3}}\left\|\tilde{W}_{i j}\right\|^{4}+\frac{3}{4} \frac{\sigma_{i 2, j}}{\varepsilon_{i j}^{4}}\left\|W_{i j}^{*}\right\|^{4}
\end{array}
$$

Furthermore

$$
\begin{array}{r}
-\sigma_{i 2, j}\left\|W_{i j}^{*}\right\|^{2} \tilde{W}_{i j}^{T} W_{i j}^{*} \leq \sigma_{i 2, j}\left\|W_{i j}^{*}\right\|\left\|\tilde{W}_{i j}^{T}\right\|\left\|W_{i j}^{*}\right\| \\
\leq \sigma_{i 2, j}\left\|\tilde{W}_{i j}\right\|^{2}\left\|W_{i j}^{*}\right\|^{2}+\frac{\sigma_{i 2, j}}{4}\left\|W_{i j}^{*}\right\|^{4}
\end{array}
$$

Substituting (78), (79) into (77), we can get

$$
\begin{gathered}
-\sigma_{i 2, j} \tilde{W}_{i j}^{T} \hat{W}_{i j} \hat{W}_{i j}^{T} \hat{W}_{i j} \leq-\sigma_{i 2, j}\left(1-\frac{9 \varepsilon_{i j}^{\frac{4}{3}}}{4}\right)\left\|\tilde{W}_{i j}\right\|^{4} \\
+\frac{3}{4} \frac{\sigma_{i 2, j}}{\varepsilon_{i j}^{4}}\left\|W_{i j}^{*}\right\|^{4}+\frac{\sigma_{i 2, j}}{4}\left\|W_{i j}^{*}\right\|^{4}
\end{gathered}
$$

where $\varepsilon_{i j} \geq 0$. we choose $\left(1-\frac{9 \varepsilon_{i j}^{\frac{4}{3}}}{4}\right) \leq 0$, hence we can get $\varepsilon_{i j} \geq\left(\frac{2}{3}\right)^{\frac{3}{2}}$.

Substituting (73), (80) into (71), we have

$$
\begin{aligned}
\dot{V}_{4} \leq \sum_{i=m, s}[ & -z_{2 i}{ }^{T} \tilde{W}_{i}^{T} \varphi_{i}\left(X_{i}\right) \\
& -\sum_{j=1}^{n} \sigma_{i 2, j}\left(4-9 \varepsilon_{i j}^{\frac{4}{3}}\right)\left(\frac{1}{2}\left\|\tilde{W}_{i j}\right\|^{2}\right)^{2} \\
& \left.-\sum_{j=1}^{n} \sigma_{i 3, j}\left(\frac{1}{2}\left\|\tilde{W}_{i j}\right\|^{2}\right)^{\frac{3}{4}}+C_{i 4}\right]
\end{aligned}
$$

where $C_{i 4}$ is positive constant as follows:

$$
\begin{aligned}
C_{i 4}= & \sum_{j=1}^{n}\left(\frac{3}{4} \frac{\sigma_{i 2, j}}{\varepsilon_{i j}^{4}}\left\|W_{i j}^{*}\right\|^{4}+\frac{\sigma_{i 2, j}}{4}\left\|W_{i j}^{*}\right\|^{4}\right. \\
& \left.+\frac{\sigma_{i 1, j}}{2}\left\|W_{i j}^{*}\right\|^{2}+\frac{\sigma_{i 3, j}^{4}}{4 \sigma_{i 1, j}^{3}}\right)
\end{aligned}
$$

In above all, substituting (53), (57), (68) and (81) into (51), we have

$$
\begin{aligned}
\dot{V} \leq & \sum_{i=m, s}\left[z_{1 i}^{T} z_{2 i}+z_{1 i}^{T} \dot{q}_{i^{\prime}}\left(t-\Delta T_{i^{\prime}}\right) \Delta \dot{T}_{i^{\prime}}-k_{i 11}\left(z_{1 i}^{T} z_{1 i}\right)^{\frac{3}{4}}\right. \\
& -k_{i 12}\left(z_{1 i}^{T} z_{1 i}\right)^{2}-k_{i 21}\left(\frac{1}{2} z_{2 i}^{T} M_{o i} z_{2 i}\right)^{\frac{3}{4}}-k_{i 22}\left(\frac{1}{2} z_{2 i}^{T} M_{o i} z_{2 i}\right)^{2} \\
& +z_{2 i}^{T}\left(\tilde{W}_{i}^{T} \varphi_{i}\left(x_{i}\right)-\Delta \dot{T}_{i^{\prime}} M_{o i}\left(q_{i}\right) f\left(z_{1 i}\right) \dot{q}_{i^{\prime}}\left(t-\Delta T_{i^{\prime}}\right)-z_{1 i}\right. \\
& \left.-\hat{D}_{i} N_{i}^{T} N_{i} z_{2 i}\right)+\frac{1}{2}\left\|\bar{\epsilon}_{i}\right\|^{2}+\tilde{D}_{i} z_{2 i}^{T} N_{i} N_{i}^{T} z_{2 i}-\mu_{i 3}\left(\frac{\tilde{D}_{i}^{2}}{2}\right)^{\frac{3}{4}} \\
& -\mu_{i 2}\left(1-\frac{9 \varepsilon_{i}^{4}}{4}\right) \tilde{D}_{i}^{4}+C_{i 3}-\sum_{j=1}^{n} \sigma_{i 2, j}\left(4-9 \varepsilon_{i j}^{\frac{4}{3}}\right)\left(\frac{1}{2}\left\|\tilde{W}_{i j}\right\|^{2}\right)^{2} \\
& \left.-z_{2 i}{ }^{T} \tilde{W}_{i}^{T} \varphi_{i}\left(x_{i}\right)-\sum_{j=1}^{n} \sigma_{i 3, j}\left(\frac{1}{2}\left\|\tilde{W}_{i j}\right\|^{2}\right)^{\frac{3}{4}}+C_{i 4}\right]
\end{aligned}
$$

After simplification, we have

$$
\begin{aligned}
\dot{V} \leq & \sum_{i=m, s}\left[-k_{i 11}\left(z_{1 i}^{T} z_{1 i}\right)^{\frac{3}{4}}+\Delta \dot{T}_{i^{\prime}} z_{2 i}^{T} N_{i}-k_{i 12}\left(z_{1 i}^{T} z_{1 i}\right)^{2}\right. \\
& -k_{i 21}\left(\frac{1}{2} z_{2 i}^{T} M_{o i} z_{2 i}\right)^{\frac{3}{4}}-k_{i 22}\left(\frac{1}{2} z_{2 i}^{T} M_{o i} z_{2 i}\right)^{2}-\hat{D}_{i} z_{2 i}^{T} N_{i}^{T} N_{i} z_{2 i} \\
& +\tilde{D}_{i} z_{2 i}^{T} N_{i} N_{i}^{T} z_{2 i}-\mu_{i 3}\left(\frac{\tilde{D}_{i}^{2}}{2}\right)^{\frac{3}{4}}-\mu_{i 2}\left(1-\frac{9 \varepsilon_{i}^{4}}{4}\right) \tilde{D}_{i}^{4} \\
& +\frac{1}{2}\left\|\bar{\epsilon}_{i}\right\|^{2}+C_{i 3}-\sum_{j=1}^{n} \sigma_{i 2, j}\left(4-9 \varepsilon_{i j}^{\frac{4}{3}}\right)\left(\frac{1}{2}\left\|\tilde{W}_{i j}\right\|^{2}\right)^{2} \\
& \left.-\sum_{j=1}^{n} \sigma_{i 3, j}\left(\frac{1}{2}\left\|\tilde{W}_{i j}\right\|^{2}\right)^{\frac{3}{4}}+C_{i 4}\right]
\end{aligned}
$$

For $\Delta \dot{T}_{i^{\prime}} z_{2 i}^{T} N_{i}$, we have

$$
\begin{aligned}
\Delta \dot{T}_{i^{\prime}} z_{2 i}^{T} N_{i} & \leq\left|\Delta \dot{T}_{i^{\prime}}\right| z_{2 i}^{T} N_{i} N_{i}^{T} z_{2 i}+\frac{1}{4}\left|\Delta \dot{T}_{i^{\prime}}\right| \\
& \leq D_{i} z_{2 i}^{T} N_{i} N_{i}^{T} z_{2 i}+\frac{1}{4} D_{i}
\end{aligned}
$$

Substituting (85) into (84), we can get

$$
\begin{aligned}
\dot{V} \leq & \sum_{i=m, s}\left[-k_{i 11}\left(z_{1 i}^{T} z_{1 i}\right)^{\frac{3}{4}}-k_{i 12}\left(z_{1 i}^{T} z_{1 i}\right)^{2}-k_{i 21}\left(\frac{1}{2} z_{2 i}^{T} M_{o i} z_{2 i}\right)^{\frac{3}{4}}\right. \\
& -k_{i 22}\left(\frac{1}{2} z_{2 i}^{T} M_{o i} z_{2 i}\right)^{2}-\mu_{i 3}\left(\frac{\tilde{D}_{i}^{2}}{2}\right)^{\frac{3}{4}}-\mu_{i 2}\left(4-9 \varepsilon_{i}^{4}\right)\left(\frac{1}{2} \tilde{D}_{i}^{2}\right)^{2} \\
& -\sum_{j=1}^{n} \sigma_{i 2, j}\left(4-9 \varepsilon_{i j}^{\frac{4}{3}}\right)\left(\frac{1}{2}\left\|\tilde{W}_{i j}\right\|^{2}\right)^{2}-\sum_{j=1}^{n} \sigma_{i 3, j}\left(\frac{1}{2}\left\|\tilde{W}_{i j}\right\|^{2}\right)^{\frac{3}{4}} \\
& \left.+C_{i}\right]
\end{aligned}
$$

where $C_{i}$ is positive constant as follows:

$$
C_{i}=\frac{1}{4} D_{i}+\frac{1}{2}\left\|\bar{\epsilon}_{i}\right\|^{2}+C_{i 3}+C_{i 4}
$$


Define

$$
\begin{aligned}
\rho_{1}= & \min _{i=m, s}\left(k_{i 11}, k_{i 21}, \mu_{i 3} \lambda_{i}^{\frac{3}{4}}, \min _{j=1,2, \ldots n} \sigma_{i 3, j}\left(\frac{1}{\lambda_{\max }\left(\Gamma_{i j}^{-1}\right)}\right)^{\frac{3}{4}}\right) \\
\rho_{2}= & \min _{i=m, s}\left(k_{i 12}, k_{i 22}, \mu_{i 2}\left(4-9 \varepsilon_{i}^{4}\right) \lambda_{i}{ }^{2},\right. \\
& \left.\min _{j=1,2, \ldots n} \sigma_{i 2, j}\left(4-9 \varepsilon_{i j}^{\frac{4}{3}}\right)\left(\frac{1}{\lambda_{\max }\left(\Gamma_{i j}^{-1}\right)}\right)^{2}\right)
\end{aligned}
$$

From Lemma 1, we can know

$$
\begin{aligned}
-\rho_{1} V^{\frac{3}{4}} \geq & \sum_{i=m, s}\left[-k_{i 11}\left(\frac{1}{2} z_{1 i}^{T} z_{1 i}\right)^{\frac{3}{4}}-k_{i 21}\left(\frac{1}{2} z_{2 i}^{T} M_{o i} z_{2 i}\right)^{\frac{3}{4}}\right. \\
& -\sigma_{i 3, j}\left(\frac{1}{\lambda_{\max }\left(\Gamma_{i j}^{-1}\right)}\right)^{\frac{3}{4}}\left(\frac{1}{2} \sum_{j=1}^{n} \tilde{W}_{i j}^{T} \Gamma_{i j}^{-1} \tilde{W}_{i j}\right)^{\frac{3}{4}} \\
& \left.-\mu_{i 3} \lambda_{i}{ }^{\frac{3}{4}}\left(\frac{1}{2 \lambda_{i}} \tilde{D}_{i}^{2}\right)^{\frac{3}{4}}\right]
\end{aligned}
$$

From Lemma 3, we can know

$$
\begin{aligned}
-\frac{\rho_{2}}{2 n+6} V^{2} & \geq \sum_{i=m, s}\left[-k_{i 11}\left(\frac{1}{2} z_{1 i}^{T} z_{1 i}\right)^{2}-k_{i 21}\left(\frac{1}{2} z_{2 i}^{T} M_{o i} z_{2 i}\right)^{2}\right. \\
& -\sigma_{i 2, j}\left(4-9 \varepsilon_{i j}^{\frac{4}{3}}\right)\left(\frac{1}{\lambda_{\max }\left(\Gamma_{i j}^{-1}\right)}\right)^{2}\left(\frac{1}{2} \sum_{j=1}^{n} \tilde{W}_{i j}^{T} \Gamma_{i j}^{-1} \tilde{W}_{i j}\right)^{2} \\
& \left.-\mu_{i 2}\left(4-9 \varepsilon_{i}^{4}\right) \lambda_{i}{ }^{2}\left(\frac{1}{2 \lambda_{i}} \tilde{D}_{i}^{2}\right)^{2}\right]
\end{aligned}
$$

Considering (86), (89) and (90), we have

$$
\dot{V} \leq-\rho_{1} V^{\frac{3}{4}}-\frac{\rho_{2}}{2 n+6} V^{2}+C
$$

where $C=C_{m}+C_{s}$.

Theorem 1: For the bilateral teleoperation systems described in (14), applying the adaptive neural network control scheme in (39) and adaptive laws in (45), if the time delay in the communication channel satisfies the Assumption 1, and the initial conditions $\left(q_{i}(0), \dot{q}_{i}(0), \hat{D}_{i}, \hat{W}_{i j}\right)$ are bounded. Then, the bilateral teleoperation systems error signals $z_{1 i}, z_{2 i}, \tilde{D}_{i}$ and $\tilde{W}_{i}$ will converge into compact sets $\Omega_{z_{1 i}}, \Omega_{z_{2 i}}, \Omega_{\tilde{D}_{i}}$ and $\Omega_{\tilde{W}_{i}}$ in fixed time, the settling time $T$ satisfies

$$
T \leq \frac{4}{\rho_{1}}+\frac{2 n+6}{(1-\beta) \rho_{2}}
$$

where $0<\beta<1, \rho_{1}$ and $\rho_{2}$ are positive constants, $n$ is a positive integer. The compact sets $\Omega_{z_{1 i}}, \Omega_{z_{2 i}}, \Omega_{\tilde{D}_{i}}$ and $\Omega_{\tilde{W}_{i}}$ have following definitions:

$$
\begin{aligned}
& \Omega_{z_{1 i}}:\left\{z_{1 i}:\left\|z_{1 i}\right\| \leq \sqrt{2 \sqrt{\frac{C(2 n+6)}{\beta \rho_{2}}}}\right\} \\
& \Omega_{z_{2 i}}:\left\{z_{2 i}:\left\|z_{2 i}\right\| \leq \sqrt{\frac{2 \sqrt{\frac{C(2 n+6)}{\beta \rho_{2}}}}{\lambda_{\min }\left(M_{o i}\right)}}\right\} \\
& \Omega_{\tilde{D}_{i}}:\left\{\tilde{D}_{i}:\left\|\tilde{D}_{i}\right\| \leq \sqrt{2 \lambda_{i} \sqrt{\frac{C(2 n+6)}{\beta \rho_{2}}}}\right\} \\
& \Omega_{\tilde{W}_{i}}:\left\{\tilde{W}_{i j}:\left\|\tilde{W}_{i j}\right\| \leq \sqrt{\frac{2 \sqrt{\frac{C(2 n+6)}{\beta \rho_{2}}}}{\lambda_{\min }\left(\Gamma_{i j}^{-1}\right)}}\right\}
\end{aligned}
$$

Proof: For the fixed-time convergence case, note that when $V^{2} \leq \frac{C(2 n+6)}{\beta \rho_{2}}$ for $0 \leq \beta \leq 1$, we have $C \leq \frac{\beta \rho_{2}}{2 n+6} V^{2}$, hence we can obtain

$$
\dot{V} \leq-\rho_{1} V^{\frac{3}{4}}-(1-\beta) \frac{\rho_{2}}{2 n+6} V^{2}
$$

Under Lemma 5, we can obtain that $V$ will converge into compact set $\left\{V: V<\sqrt{\frac{C(2 n+6)}{\beta \rho_{2}}}\right\}$ in fixed time, the settling time $T$ satisfies

$$
T \leq \frac{4}{\rho_{1}}+\frac{2 n+6}{(1-\beta) \rho_{2}}
$$

$V$ converging into compact set $\left\{V: V<\sqrt{\frac{C(2 n+6)}{\beta \rho_{2}}}\right\}$ in fixed time promises that $\frac{1}{2} z_{1 i}^{T} z_{1 i} \leq \sqrt{\frac{C(2 n+6)}{\beta \rho_{2}}}$, hence we have $\left\|z_{1 i}\right\| \leq \sqrt{2 \sqrt{\frac{C(2 n+6)}{\beta \rho_{2}}}}$, and $z_{1 i}$ will converge into compact set $\Omega_{z_{1 i}}$ in fixed time with guaranteed settling time estimated as $T$. In the same manner, we can easily prove that $z_{2 i}, \tilde{D}_{i}$ and $\tilde{W}_{i}$ will converge into compact sets $\Omega_{z_{2 i}}, \Omega_{\tilde{D}_{i}}, \Omega_{\tilde{W}_{i}}$. Then, the proof of Theorem 1 is completed.

\section{Simulation}

In this section, we use MATLAB software to validate the feasibility of proposed fixed-time controller in teleoperation systems. Dynamics are considered to be two degrees of freedom (DOF) in master side and slave side. The nominal values of master robot and slave robot by reference to [56] are shown in TABLE I.

TABLE I

NOMINAL VALUES OF MASTER AND SLAVE ROBOT

\begin{tabular}{|c|c|c|}
\hline Parameter & Value & Physical Description \\
\hline$m_{i 1}$ & $2.00 \mathrm{~kg}$ & Mass of linkage 1 \\
\hline$m_{i 2}$ & $0.85 \mathrm{~kg}$ & Mass of linkage 2 \\
\hline$l_{i 1}$ & $0.35 \mathrm{~m}$ & Length of linkage 1 \\
\hline$l_{i 2}$ & $0.31 \mathrm{~m}$ & Length of linkage 2 \\
\hline$I_{i 1}$ & $6.125 \times 10^{-2} \mathrm{kgm}^{2}$ & Inertia of linkage 1 \\
\hline$I_{i 2}$ & $2.042 \times 10^{-2} \mathrm{kgm}^{2}$ & Inertia of linkage 2 \\
\hline
\end{tabular}

The dynamic description of systems are shown as follow:

$$
\begin{aligned}
& M_{q i}\left(q_{i}\right)=\left[\begin{array}{cc}
p_{i 1}+p_{i 2}+2 p_{i 3} \cos \left(q_{i 2}\right) & p_{i 2}+p_{i 3} \cos \left(q_{i 2}\right) \\
p_{i 2}+p_{i 3} \cos \left(q_{i 2}\right) & p_{i 2}
\end{array}\right] \\
& C_{q i}\left(q_{i}, \dot{q}_{i}\right)=\left[\begin{array}{cc}
-p_{i 3} \dot{q}_{i 2} \sin \left(q_{i 2}\right) & -p_{i 3}\left(\dot{q}_{i 1}+\dot{q}_{i 2}\right) \sin \left(q_{i 2}\right) \\
p_{i 3} \dot{q}_{i 1} \sin \left(q_{i 2}\right) & 0
\end{array}\right] \\
& G_{q i}\left(q_{i}\right)=\left[\begin{array}{c}
p_{i 4} g \cos \left(q_{i 1}\right)+p_{i 5} g \cos \left(q_{i 1}+q_{i 2}\right) \\
p_{i 5} g \cos \left(q_{i 1}+q_{i 2}\right)
\end{array}\right] \\
& H_{i 1}=l_{i 1} \cos \left(q_{i 1}\right)+l_{i 2} \cos \left(q_{i 1}+q_{i 2}\right) \\
& H_{i 2}=l_{i 1} \sin \left(q_{i 1}\right)+l_{i 2} \sin \left(q_{i 1}+q_{i 2}\right) \\
& H_{i}=\left[\begin{array}{ll}
H_{i 1} & H_{i 2}
\end{array}\right] \\
& J_{i 11}=-l_{i 1} \sin \left(q_{i 1}\right)-l_{i 2} \sin \left(q_{i 1}+q_{i 2}\right) \\
& J_{i 12}=-l_{i 2} \sin \left(q_{i 1}+q_{i 2}\right) \\
& J_{i 21}=l_{i 1} \cos \left(q_{i 1}\right)+l_{i 2} \cos \left(q_{i 1}+q_{i 2}\right) \\
& J_{i 22}=l_{i 2} \cos \left(q_{i 1}+q_{i 2}\right) \\
& J_{i}\left(q_{i}\right)=\left[\begin{array}{ll}
J_{i 11} & J_{i 12} \\
J_{i 21} & J_{i 22}
\end{array}\right]
\end{aligned}
$$

The values of $p_{i 1}, p_{i 2}, p_{i 3}, p_{i 4}, p_{i 5}$ can be calculated as: $p_{i 1}=$ $m_{i 1} l_{i c 1}^{2}+m_{i 2} l_{i 1}^{2}+I_{i 1}, p_{i 2}=m_{i 2} l_{i c 2}^{2}+I_{i 2}, p_{i 3}=m_{i 2} l_{i 1} l_{i c 2}$, 
$p_{i 4}=m_{i} 1 l_{i c 2}+m_{i 2} l_{i 1}, p_{i 5}=m_{i 2} l_{i c 2}$. And we set the operator and environment parameters as: $M_{h}=\operatorname{diag}[0.1,0.1]$, $M_{e}=\operatorname{diag}[0.2,0.2], B_{h}=\operatorname{diag}[2,2], B_{e}=\operatorname{diag}[2,2]$, $K_{h}=\operatorname{diag}[5,5]$ and $K_{e}=\operatorname{diag}[5,5]$.

We have a hypothesis that the uncertainty parts of bilateral teleoperation can be denoted as: $\Delta M_{i}\left(q_{i}\right)=0.2 M_{o i}\left(q_{i}\right)$, $\Delta C_{i}\left(q_{i}, \dot{q}_{i}\right)=0.2 C_{o i}\left(q_{i}, \dot{q}_{i}\right), \Delta G_{i}\left(q_{i}\right)=0.2 G_{o i}\left(q_{i}\right)$.

We set initial positions of master and slave robots as follows: $q_{m 1}(0)=q_{m 2}(0)=0.1 \mathrm{rad}, \dot{q}_{m 1}(0)=\dot{q}_{m 2}(0)=0$ $\mathrm{rad} / \mathrm{s}, q_{s 1}(0)=q_{s 2}(0)=-0.1 \mathrm{rad}, \dot{q}_{s 1}(0)=\dot{q}_{s 2}(0)=0$ $\mathrm{rad} / \mathrm{s} . \Delta T_{m}=0.3+0.02 \sin (2 t)+0.03 \sin (3 t)+0.05 \sin (5 t) \mathrm{s}$, $\Delta T_{s}=0.3+0.01 \sin (t)+0.03 \sin (3 t)+0.06 \sin (6 t) \mathrm{s}$ in Fig. 3. We assume that $t \in\left(0, t_{f}\right)$ and $t_{f}=45 \mathrm{~s}$. We have an hypothesis that the human operator force $f_{h}$ which is applied to master robot exists in $\mathrm{x}$ direction, we assign $f_{h}$ as $-8 \mathrm{~N}$ from 10 to $15 \mathrm{~s}$ and $5 \mathrm{~N}$ from 30 to $35 \mathrm{~s}$, which is shown in Fig. 4. The component of $f_{h}$ in y direction is $0 \mathrm{~N}$, environment force applied to slave robot in both $\mathrm{x}$ and $\mathrm{y}$ directions are $0 \mathrm{~N}$.
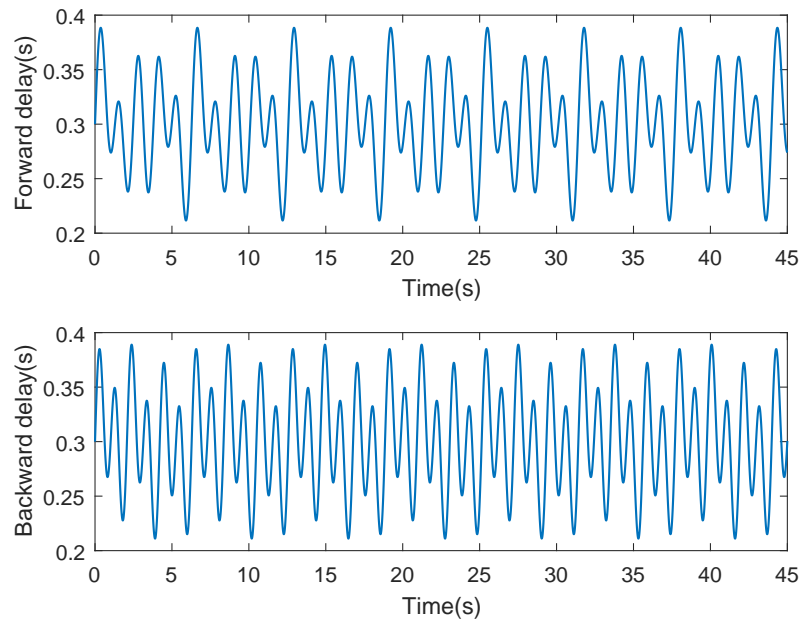

Fig. 3. Communication delays in forward channel and backward channel

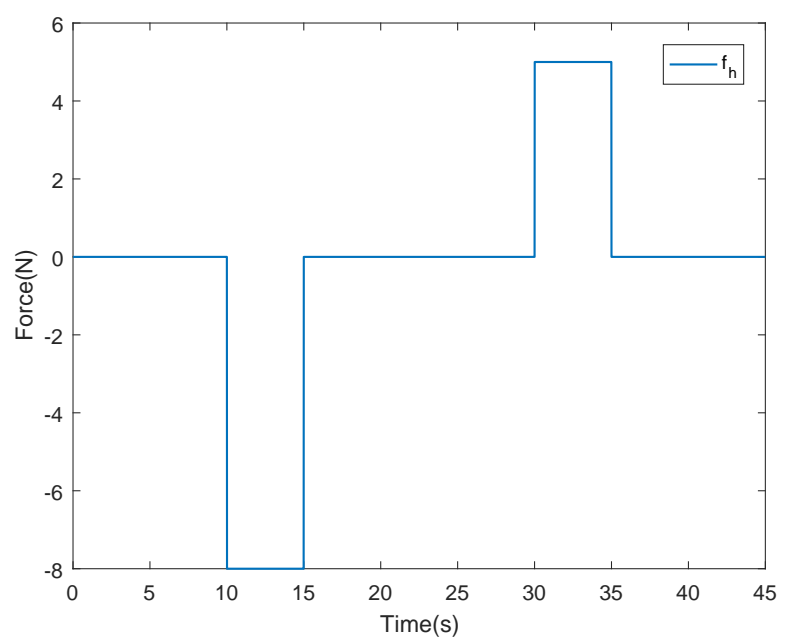

Fig. 4. $f_{h}$ at $\mathrm{x}$ direction

The process of simulation can be briefly explained as follows: Master and slave robots exists displacement differences in the initial states of teleoperation system. When there is no external force, slave robot can follow master robot and converge to the equilibrium point. At $10 \mathrm{~s}$, the master robot receives force from the human operator and begin to move. The slave robot will follow the master robot. After $5 \mathrm{~s}$ the force of human operator is vanish then system synchronization errors can converge to a small neighborhood around zero in fixed time. At 30s the master robot receive opposite force in $\mathrm{x}$ direction, the teleoperation system also keep stability and synchronization errors converge to a small neighborhood around zero in fixed time.

We divide simulations into two parts: In the first part, we will perform the analysis of proposed adaptive fixedtime control scheme. In the second part, the proposed control method will be compared with finite-time control method, which is often used in teleoperation systems to promise the settling time of system states.

\section{A. Performed analysis of proposed Adaptive fixed-time control}

The parameters of proposed controller are chosen in Table II.

TABLE II

CONTROLLER PARAMETERS

\begin{tabular}{|c|c|c|c|}
\hline Master & Value & Slave & Value \\
\hline$k_{m 11}$ & 1.5 & $k_{s 11}$ & 1.1 \\
\hline$k_{m 12}$ & 2 & $k_{s 12}$ & 1 \\
\hline$k_{m 21}$ & 2 & $k_{s 21}$ & 2 \\
\hline$k_{m 22}$ & 2 & $k_{s 22}$ & 1 \\
\hline$\sigma_{m 1}$ & 0.005 & $\sigma_{s 1}$ & 0.01 \\
\hline$\sigma_{m 2}$ & 0.005 & $\sigma_{s 2}$ & 0.01 \\
\hline$\Gamma_{m}$ & $\operatorname{diag}[1,1]$ & $\Gamma_{s}$ & $\operatorname{diag}[1,1]$ \\
\hline$\mu_{m 1}$ & 0.001 & $\gamma_{s 1}$ & 0.001 \\
\hline$\mu_{m 2}$ & 0.001 & $\mu_{s 2}$ & 0.001 \\
\hline$\lambda_{m}$ & 1 & $\lambda_{s}$ & 1 \\
\hline
\end{tabular}

Base on the selected controller, the simulation results are shown in Figs. 5-10. The tracking performance under adaptive fixed-time control is shown in Fig. 5, from the figure we can clearly see that slave robot can follow master robot in both two revolute joints. The position synchronization tracking errors $z_{1 m}$ and $z_{1 \mathrm{~s}}$ are shown in Fig. 6, the tracking errors converge to a small neighborhood around zero in fixed time. The second errors variables $z_{2 m}$ and $z_{2 s}$ are shown in Fig. 7. Torque control inputs are given in Fig. 8, the effect of system time delay results in oscillations of torque control input signals. Fig. 9 shows the norm for neural network estimation weight value. Fig. 10 shows estimation value of the time-varying delay upper bound. It is obviously that $\left\|\hat{W}_{i}\right\|$ and $\left\|\hat{D}_{i}\right\|$ are bounded, which means $\left\|\tilde{W}_{i}\right\|$ and $\left\|\tilde{D}_{i}\right\|$ are also bounded.

The results in first part of simulation prove that the proposed control method is feasible and achieves high tracking performance.

\section{B. Comparison with finite-time control}

In order to verify the effectiveness of adaptive fixedtime control, we compare the proposed control method with 

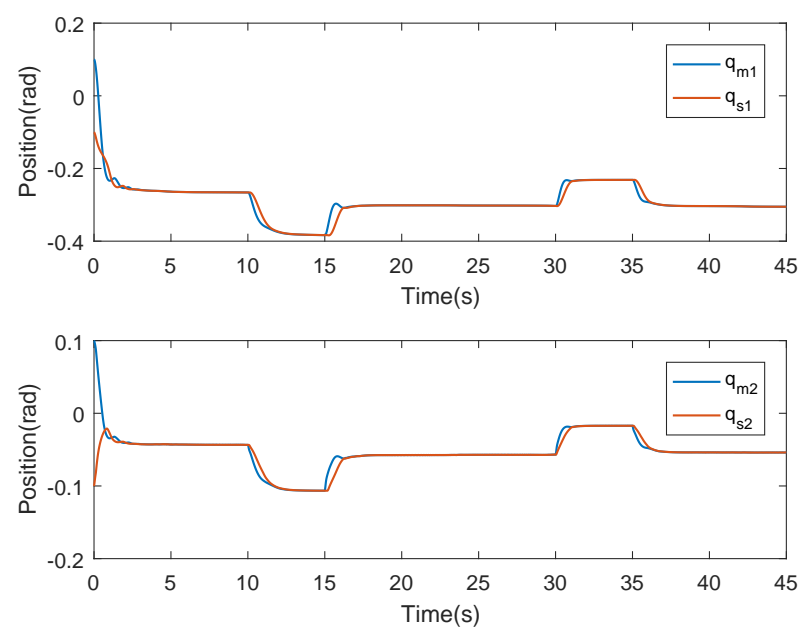

Fig. 5. Tracking performance under adaptive fixed-time control.
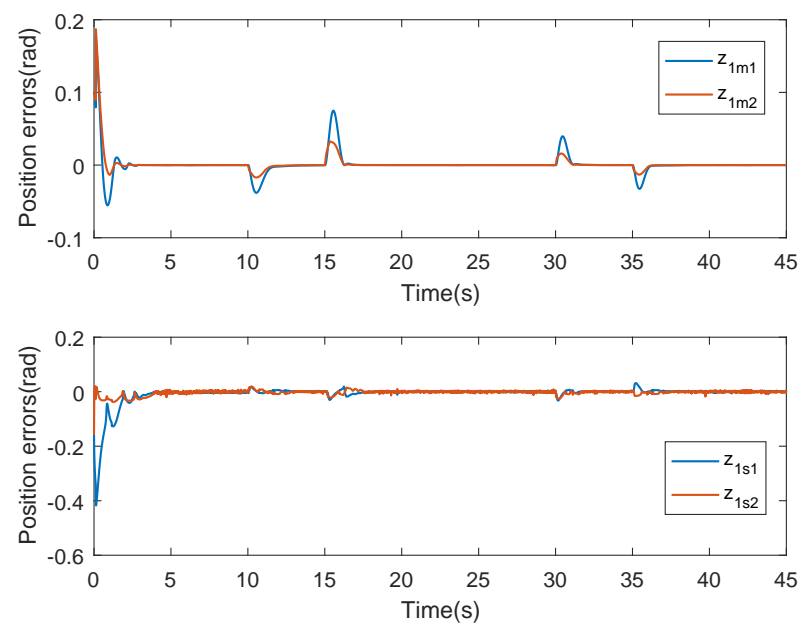

Fig. 6. Synchronization tracking errors under adaptive fixed-time control.
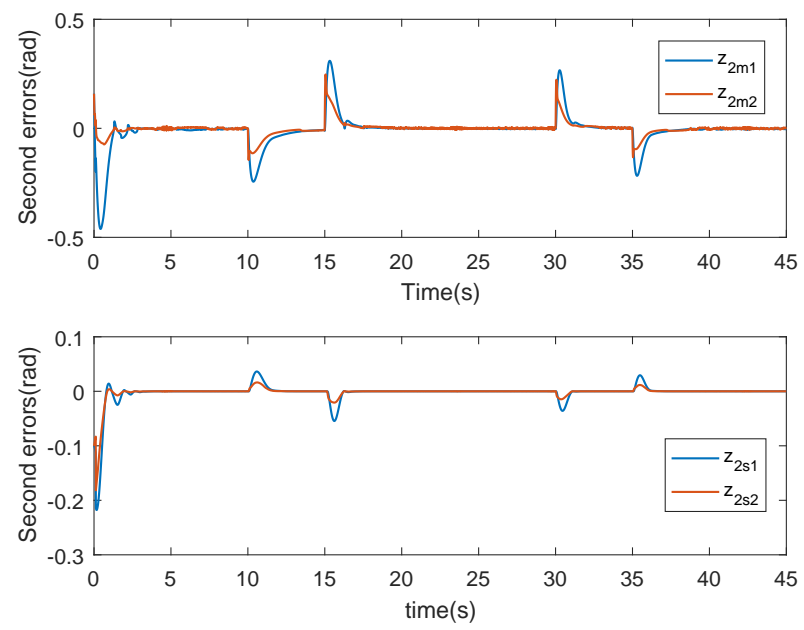

Fig. 7. Second error variables $z_{2 m}$ and $z_{2 s}$ under adaptive fixed-time control.
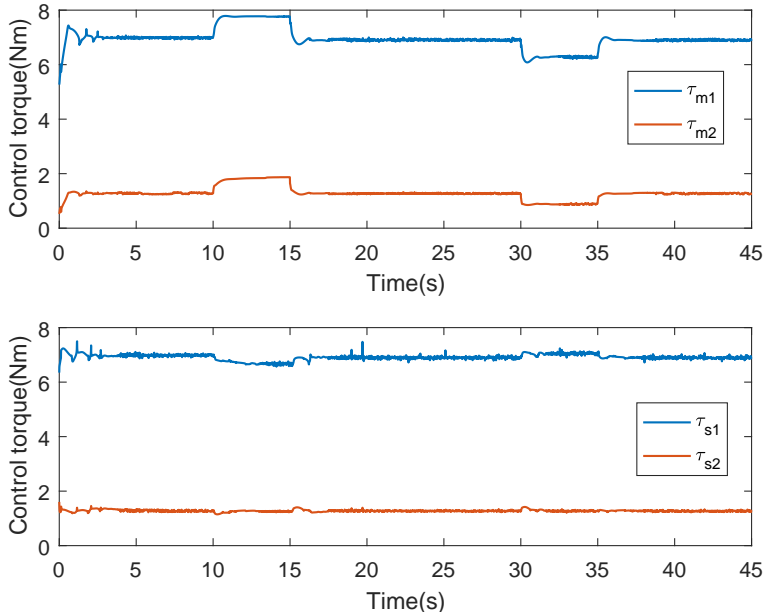

Fig. 8. Control inputs under the adaptive fixed-time control.
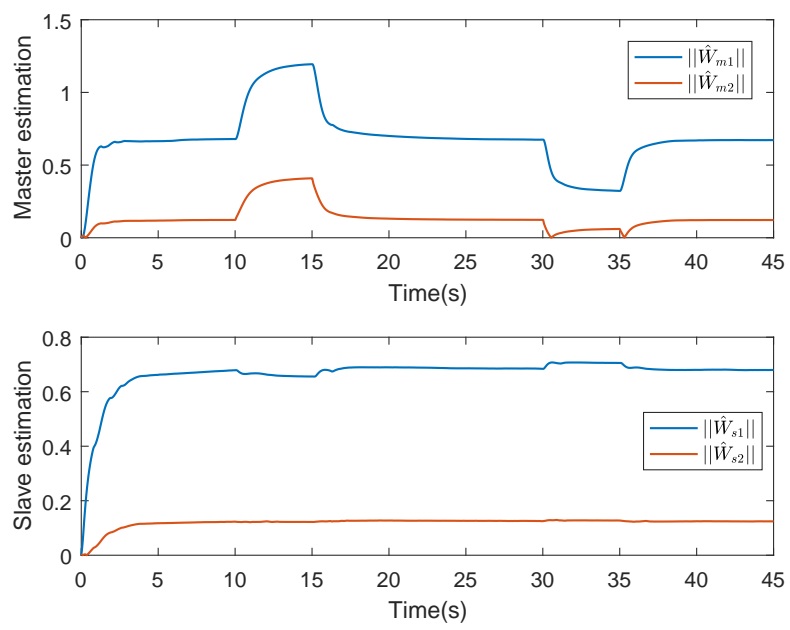

Fig. 9. Norm for neural network estimation weight value.
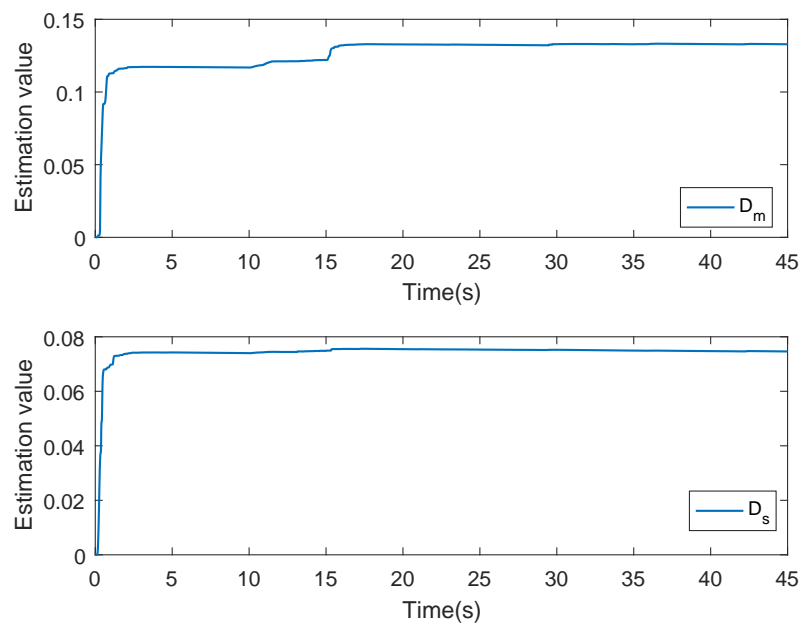

Fig. 10. Estimation value of the time-varying delay upper bound. 
adaptive finite-time control design proposed in [64]. In [64], the author propose an auxiliary compensation filter and the compensation adaptive update laws for position tracking in teleoperation systems, the study on convergence time is similar to this paper. The results of comparison are analyzed as follows:

The finite-time controller design and adaptive laws are given by [64]. Figs. 11-12 show the comparison of synchronization errors between proposed controller with finite-time controller. The consequences indicate that these two method can converge the synchronization errors to zero. However, the performance of proposed control scheme has the lower overshoot and faster convergence rate. The settling time of proposed control method and finite-time control strategy are presented in TABLE III. The finite-time control method will cause jitter due to the existence of the sliding surface. Therefore, fixed-time method can get higher tracking performance than finite-time method.

TABLE III

SETTLING TIME COMPARISON WITH FINITE-TIME CONTROL

\begin{tabular}{|c|c|c|}
\hline Position error & Fixed-time control & Finite-time control \\
\hline$z_{1 m 1}$ & $2.32 \mathrm{~s}$ & $5.30 \mathrm{~s}$ \\
\hline$z_{1 m 2}$ & $2.34 \mathrm{~s}$ & $4.46 \mathrm{~s}$ \\
\hline$z_{1 \mathrm{~s} 1}$ & $2.80 \mathrm{~s}$ & $3.80 \mathrm{~s}$ \\
\hline$z_{1 \mathrm{~s} 2}$ & $2.78 \mathrm{~s}$ & $3.20 \mathrm{~s}$ \\
\hline
\end{tabular}
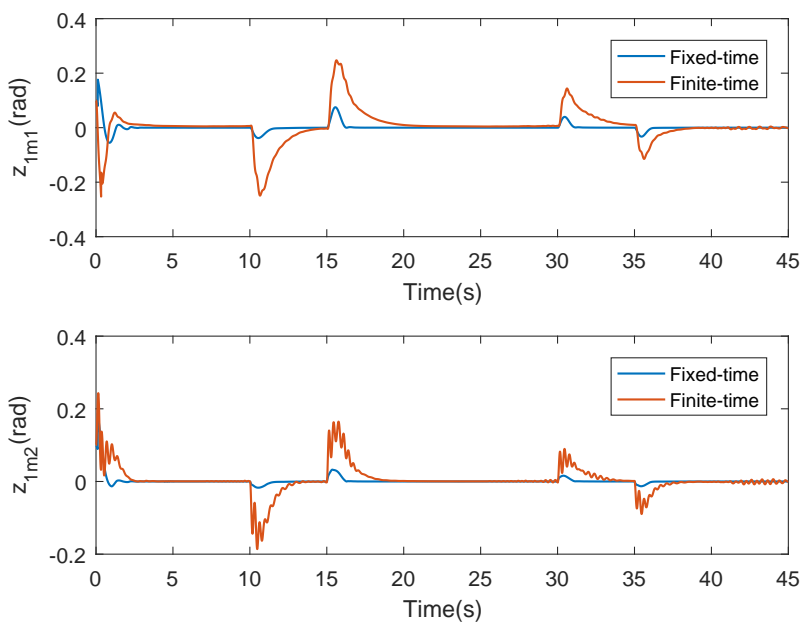

Fig. 11. The comparison of position synchronization errors $z_{1 m 1}$ and $z_{1 m 2}$ between fixed-time control and finite-time control.

\section{EXPERIMENT STUDY}

In this section, we build a teleoperation system platform to verify the effectiveness of proposed adaptive fixed-time control scheme. Phantom Omni haptic device is considered as master robot, we connect it to MATLAB Simulink environment through Quarc 2.6 platform developed by Quanser. In the slave side, the KINOVA $\mathrm{JACO}^{2}$ manipulator is considered as the slave robot in teleoperation system platform. The slave controller is established with MATLAB software. The framework of teleoperation system platform is shown in Fig. 13. The master and slave robot have 6 degrees of freedom $\left(\omega_{1}\right.$
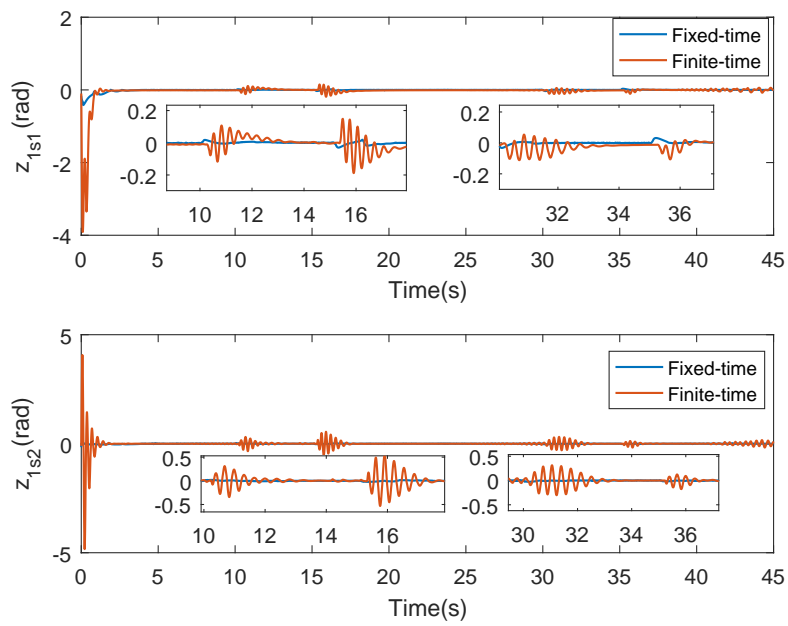

Fig. 12. The comparison of position synchronization errors $z_{1 s 1}$ and $z_{1 s 2}$ between fixed-time control and finite-time control.

to $\omega_{6}$ in master robot and $\theta_{1}$ to $\theta_{6}$ in slave robot are shown in Fig. 12). In the experiment, we choose $\omega_{1}, \omega_{2}$ and $\theta_{1}, \theta_{2}$ in slave robot as our experimental objects.

Remark 7: In the experiment, User Datagram Protocol (UDP) technology in local area network is applied in data transmission. We found that the delay of UDP transmission in the LAN is less than $2 \mathrm{~ms}$. This delay is too small to effectively influence the result in the experiment, hence we have added sinusoidal-like time-varying delay in communication channel which is far larger than time delay generated by UDP communication. When we set data throughput properly, the delay caused by UDP communication has very little impact on the experiment, and the Assumption 1 can be satisfied in the experiment.

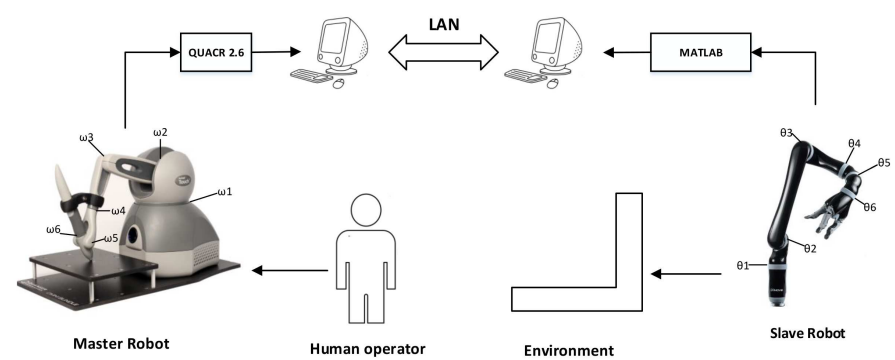

Fig. 13. The framework of teleoperation system platform.

The process of experiment can be briefly described as follows: Human operator moves the joystick of master robot and generate a series of random trajectories. The trajectory information is transmitted to the slave side through the communication channel. Then, the designed controller control the slave robot follow the master robot's movement. The bilateral teleoperation operation diagram is shown in Fig. 14. The communication latency is shown in Fig. 15. The designed adaptive fixed-time control method will be be compared to adaptive finite-time control design in [64]. Controller parameter selection in experiment is shown in TABLE IV:

The tracking performances under adaptive fixed-time control scheme and adaptive finite-time control method are shown 


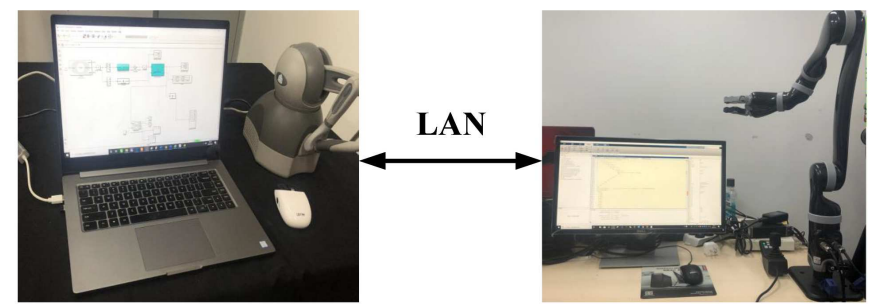

Master side

Slave side

Fig. 14. Bilateral teleoperation operation diagram.
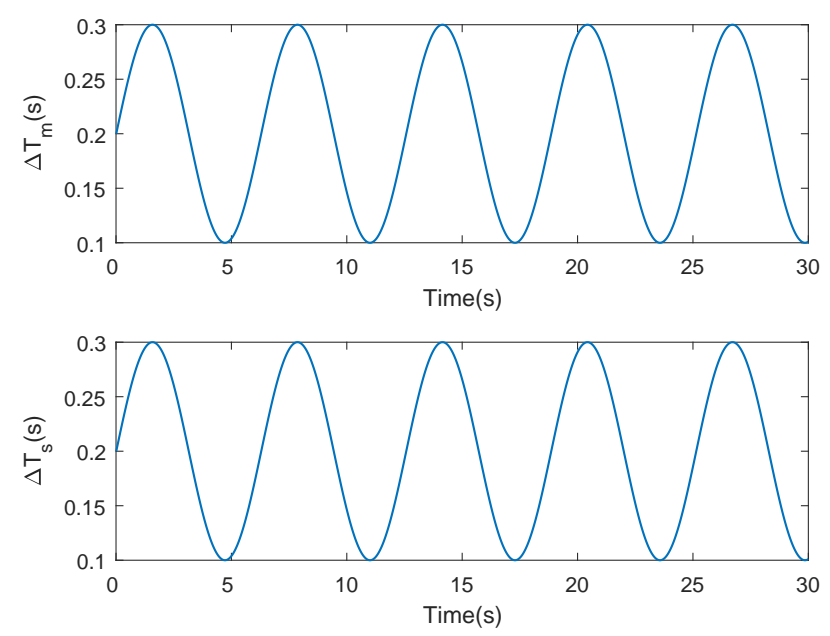

Fig. 15. Communication channel delay.

in Fig. 16 and 17. The comparison of synchronization errors are shown in Fig. 18. As we can see, the proposed adaptive fixed-time control method obtain less convergence time, smaller overshoot and steady-state error in the comparison experiment, which show that the adaptive fixed-time controller can obtain higher quality tracking performance than fixed-time control scheme.

\section{CONCLUSION}

In this paper, considering time delay in bilateral teleoperation systems, an adaptive fixed-time control has been

TABLE IV

CONTROLLER PARAMETERS IN FIXED-TIME CONTROL

\begin{tabular}{|c|c|c|c|}
\hline Master & Value & Slave & Value \\
\hline$k_{m 11}$ & 2 & $k_{s 11}$ & 2 \\
\hline$k_{m 12}$ & 1.2 & $k_{s 12}$ & 1 \\
\hline$k_{m 21}$ & 5 & $k_{s 21}$ & 5 \\
\hline$k_{m 22}$ & 3 & $k_{s 22}$ & 3 \\
\hline$\sigma_{m 1}$ & 0.01 & $\sigma_{s 1}$ & 0.01 \\
\hline$\sigma_{m 2}$ & 0.01 & $\sigma_{s 2}$ & 0.01 \\
\hline$\Gamma_{m}$ & $\operatorname{diag}[1,1]$ & $\Gamma_{s}$ & $\operatorname{diag}[1,1]$ \\
\hline$\mu_{m 1}$ & 0.01 & $\gamma_{s 1}$ & 0.01 \\
\hline$\mu_{m 2}$ & 0.01 & $\mu_{s 2}$ & 0.01 \\
\hline$\lambda_{m}$ & 1 & $\lambda_{s}$ & 1 \\
\hline
\end{tabular}
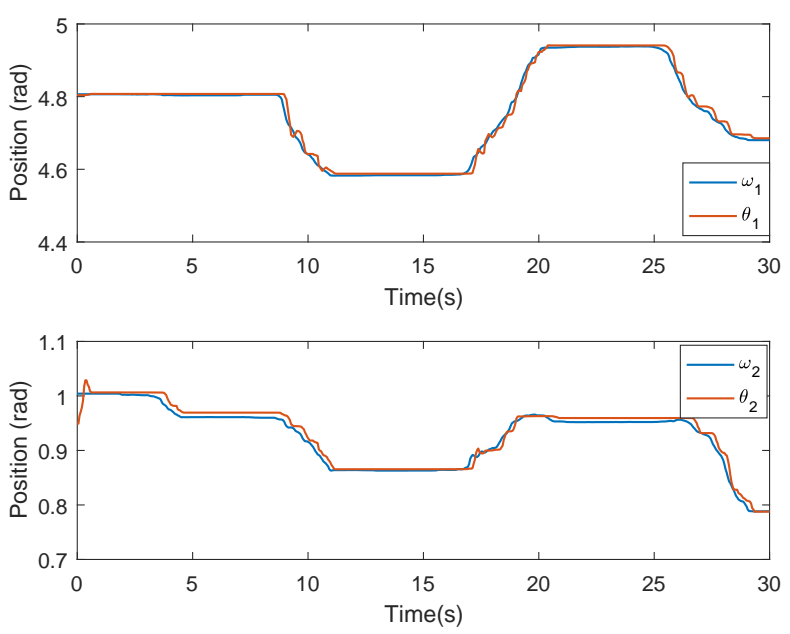

Fig. 16. Tracking performance under adaptive fixed-time control.
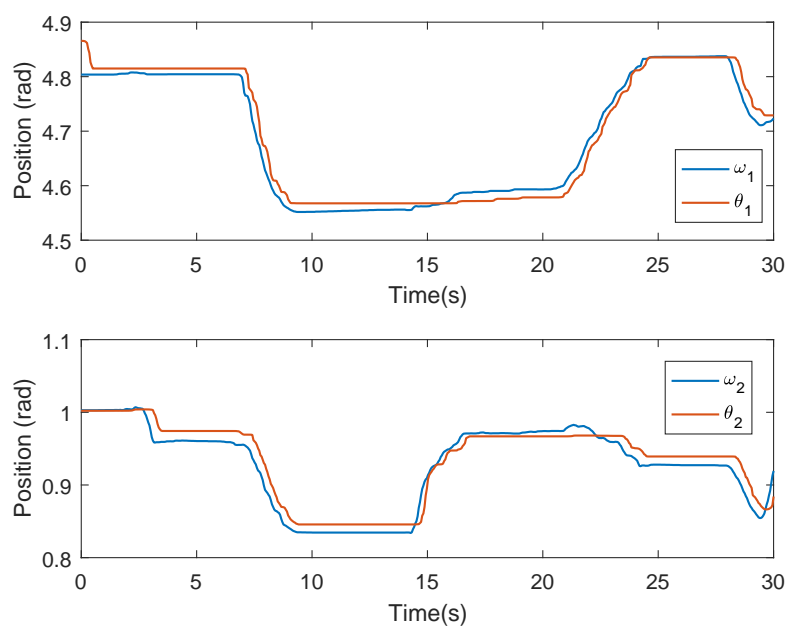

Fig. 17. Tracking performance under adaptive finite-time control.
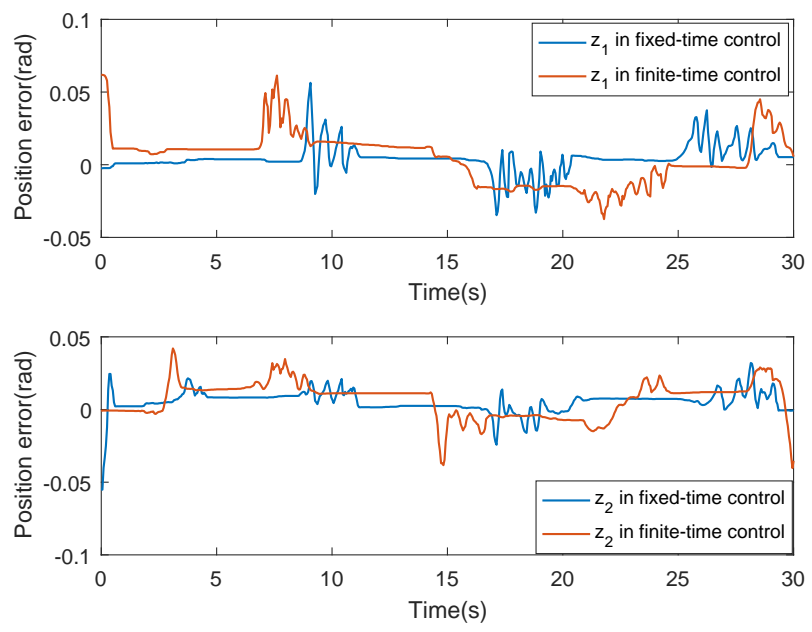

Fig. 18. Comparison of errors between adaptive fixed-time control and adaptive finite-time control. 
proposed. RBFNNs method has been used to estimate uncertain dynamic parameters of the bilateral systems. Under our proposed method, the bilateral teleoperation systems can achieve stability. The errors of systems can converge to a small neighborhood around zero in fixed time. The robustness and convergence precision of system have been improved. In simulation section and experiment study section, we have validated the feasibility of proposed method and we have compared it with adaptive finite-time control algorithm. The results show that adaptive fixed-time controller can obtain high quality tracking performances. In the future, we will discuss more general time delay cases, such as segmented communication delays labeled with sampled-data [24], etc.

\section{REFERENCES}

[1] P. F. Hokayem and M. W. Spong, "Bilateral teleoperation: An historical survey," Automatica, vol. 42, no. 12, pp. 2035-2057, 2006.

[2] G. Niemeyer and J.-J. E. Slotine, "Telemanipulation with time delays," The International Journal of Robotics Research, vol. 23, no. 9, pp. 873890, 2004

[3] W.-K. Yoon, T. Goshozono, H. Kawabe, M. Kinami, Y. Tsumaki, M. Uchiyama, M. Oda, and T. Doi, "Model-based space robot teleoperation of ets-vii manipulator," IEEE Transactions on Robotics and Automation, vol. 20, no. 3, pp. 602-612, 2004.

[4] K. Yoshida, "Achievements in space robotics," IEEE Robotics \& Automation Magazine, vol. 16, no. 4, pp. 20-28, 2009.

[5] H. Saafi, M. A. Laribi, S. Zeghloul, and M. Arsicault, "On the development of a new master device used for medical tasks," Journal of Mechanisms and Robotics, vol. 10, no. 4, p. 044501, 2018.

[6] K. Qian, A. Song, J. Bao, and H. Zhang, "Small teleoperated robot for nuclear radiation and chemical leak detection," International Journal of Advanced Robotic Systems, vol. 9, no. 3, p. 70, 2012.

[7] O. Khatib, X. Yeh, G. Brantner, B. Soe, B. Kim, S. Ganguly, H. Stuart, S. Wang, M. Cutkosky, A. Edsinger, et al., "Ocean one: A robotic avatar for oceanic discovery," IEEE Robotics \& Automation Magazine, vol. 23, no. 4, pp. 20-29, 2016.

[8] Y. Zhao and J. Xu, "Effects of delayed feedback control on nonlinear vibration absorber system," Journal of Sound and Vibration, vol. 308, no. 1, pp. 212-230, 2007.

[9] X. Zhang, Q. Han, and D. Han, "Effects of small time-delays on dynamic output feedback control of offshore steel jacket structures," Journal of Sound and Vibration, vol. 330, no. 16, pp. 3883-3900, 2011.

[10] B.-L. Zhang, Q.-L. Han, X.-M. Zhang, and X. Yu, "Sliding mode control with mixed current and delayed states for offshore steel jacket platforms," IEEE Transactions on Control Systems Technology, vol. 22, no. 5, pp. 1769-1783, 2014.

[11] X. Zhang, Q. Han, X. Ge, and D. Ding, "An overview of recent developments in lyapunovckrasovskii functionals and stability criteria for recurrent neural networks with time-varying delays," Neurocomputing, vol. 313 , pp. $392-401,2018$.

[12] A. Khalil, Z. Rajab, A. Alfergani, and O. Mohamed, "The impact of the time delay on the load frequency control system in microgrid with plugin-electric vehicles," Sustainable Cities and Society, vol. 35, pp. 365$377,2017$.

[13] N. Sharma and A. K. Gupta, "Impact of time delay on the dynamics of seir epidemic model using cellular automata," Physica A: Statistical Mechanics and its Applications, vol. 471, pp. 114-125, 2017.

[14] X. E. Zhao and B. Hu, "The impact of time delay in a tumor model," Nonlinear Analysis: Real World Applications, vol. 51, p. 103015, 2020.

[15] Z. Li, Z. Chen, J. Fu, and C. Sun, "Direct adaptive controller for uncertain mimo dynamic systems with time-varying delay and deadzone inputs," Automatica, vol. 63, pp. 287-291, 2016.

[16] T. B. Sheridan, "Space teleoperation through time delay: Review and prognosis," IEEE Transactions on Robotics and Automation, vol. 9 no. 5, pp. 592-606, 1993

[17] R. J. Anderson and M. W. Spong, "Bilateral control of teleoperators with time delay," IEEE Transactions on Automatic Control, vol. 34, no. 5 , pp. 494-501, 1989.

[18] D. A. Lawrence, "Stability and transparency in bilateral teleoperation," IEEE Transactions on Robotics and Automation, vol. 9, no. 5, pp. 624637, 1993

[19] D. Lee and M. W. Spong, "Passive bilateral teleoperation with constant time delay," IEEE Transactions on Robotics, vol. 22, no. 2, pp. 269-281, 2006.
[20] G. Niemeyer and J.-J. Slotine, "Stable adaptive teleoperation," IEEE Journal of Oceanic Engineering, vol. 16, no. 1, pp. 152-162, 1991.

[21] E. Nuño, R. Ortega, N. Barabanov, and L. Basañez, "A globally stable pd controller for bilateral teleoperators," IEEE Transactions on Robotics, vol. 24 , no. 3, pp. 753-758, 2008.

[22] E. Nuno, I. Sarras, and L. Basanez, "Consensus in networks of nonidentical euler-lagrange systems using $\mathrm{p}+\mathrm{d}$ controllers," IEEE Transactions on Robotics, vol. 29, no. 6, pp. 1503-1508, 2013.

[23] J. Yan and S. E. Salcudean, "Teleoperation controller design using $\mathrm{h} / \mathrm{sub} / \mathrm{spl}$ infin//-optimization with application to motion-scaling," IEEE Transactions on Control Systems Technology, vol. 4, no. 3, pp. 244-258, 1996.

[24] D. Zhang, Q. L. Han, and X. M. Zhang, "Network-based modeling and proportionalcintegral control for direct-drive-wheel systems in wireless network environments," IEEE Transactions on Cybernetics, vol. 50 , no. 6, pp. 2462-2474, 2020.

[25] N. Chopra, M. W. Spong, and R. Lozano, "Synchronization of bilateral teleoperators with time delay," Automatica, vol. 44, no. 8, pp. 2142 2148,2008

[26] S. Tong, S. Sui, and Y. Li, "Fuzzy adaptive output feedback control of mimo nonlinear systems with partial tracking errors constrained," IEEE Transactions on Fuzzy Systems, vol. 23, no. 4, pp. 729-742, 2015.

[27] H. Wang, P. X. Liu, and S. Liu, "Adaptive neural synchronization control for bilateral teleoperation systems with time delay and backlashlike hysteresis," IEEE Transactions on Cybernetics, vol. 47, no. 10, pp. 3018-3026, 2017.

[28] C. Yang, Y. Jiang, W. He, J. Na, Z. Li, and B. Xu, "Adaptive parameter estimation and control design for robot manipulators with finite-time convergence," IEEE Transactions on Industrial Electronics, vol. 65, no. 10 , pp. 8112-8123, 2018.

[29] H. Li, S. Zhao, W. He, and R. Lu, "Adaptive finite-time tracking control of full state constrained nonlinear systems with dead-zone," Automatica, vol. 100, pp. 99-107, 2019

[30] X. Liu, J. Lam, W. Yu, and G. Chen, "Finite-time consensus of multiagent systems with a switching protocol," IEEE Transactions on Neural Networks, vol. 27, no. 4, pp. 853-862, 2016.

[31] Y. Yang, "A time-specified nonsingular terminal sliding mode control approach for trajectory tracking of robotic airships," Nonlinear Dynamics, vol. 92, no. 3, pp. 1359-1367, 2018.

[32] A. Polyakov, "Nonlinear feedback design for fixed-time stabilization of linear control systems," IEEE Transactions on Automatic Control, vol. 57 , no. 8, pp. 2106-2110, 2012.

[33] Z. Zuo, Q.-L. Han, B. Ning, X. Ge, and X.-M. Zhang, "An overview of recent advances in fixed-time cooperative control of multiagent systems," IEEE Transactions on Industrial Informatics, vol. 14, no. 6, pp. 23222334, 2018.

[34] A. Muralidharan, R. Pedarsani, and P. Varaiya, "Analysis of fixedtime control," Transportation Research Part B: Methodological, vol. 73, pp. 81-90, 2015.

[35] X. Jin, "Adaptive fixed-time control for mimo nonlinear systems with asymmetric output constraints using universal barrier functions," IEEE Transactions on Automatic Control, 2018.

[36] W. He, T. Wang, X. He, L.-J. Yang, and O. Kaynak, "Dynamical modeling and boundary vibration control of a rigid-flexible wing system," IEEE/ASME Transactions on Mechatronics, 2020.

[37] W. He, X. Mu, L. Zhang, and Y. Zou, "Modeling and trajectory tracking control for flapping-wing micro aerial vehicles," IEEE/CAA Journal of Automatica Sinica, 2020

[38] W. He, T. Meng, X. He, and C. Sun, "Iterative learning control for a flapping wing micro aerial vehicle under distributed disturbances," IEEE transactions on cybernetics, vol. 49, no. 4, pp. 1524-1535, 2018.

[39] Z. Li, B. Huang, A. Ajoudani, C. Yang, C.-Y. Su, and A. Bicchi, "Asymmetric bimanual control of dual-arm exoskeletons for humancooperative manipulations," IEEE Transactions on Robotics, vol. 34, no. 1, pp. 264-271, 2017.

[40] S. Zhang, P. Yang, L. Kong, W. Chen, Q. Fu, and K. Peng, "Neural networks-based fault tolerant control of a robot via fast terminal sliding mode," IEEE Transactions on Systems, Man, and Cybernetics: Systems, 2019.

[41] S. Zhang, R. Liu, K. Peng, and W. He, "Boundary output feedback control for a flexible two-link manipulator system with high-gain observers," IEEE Transactions on Control Systems Technology, 2019.

[42] K. S. Narendra and K. Parthasarathy, "Identification and control of dynamical systems using neural networks," IEEE Transactions on Neural Networks, vol. 1, no. 1, pp. 4-27, 1990.

[43] S. Haykin, Neural networks: a comprehensive foundation. Prentice Hall PTR, 1994. 
[44] W. He, Z. Li, Y. Dong, and T. Zhao, "Design and adaptive neural network control for an upper limb robotic exoskeleton in presence of input saturation," IEEE Transactions on Neural Networks and Learning Systems, vol. 30, no. 1, pp. 97-108, 2019.

[45] S.-L. Dai, M. Wang, and C. Wang, "Neural learning control of marine surface vessels with guaranteed transient tracking performance," IEEE Transactions on Industrial Electronics, vol. 63, no. 3, pp. 1717-1727, 2016.

[46] Z. Li, W. Yuan, Y. Chen, F. Ke, X. Chu, and C. P. Chen, "Neuraldynamic optimization-based model predictive control for tracking and formation of nonholonomic multirobot systems," IEEE Transactions on Neural Networks and Learning Systems, vol. 29, no. 12, pp. 6113-6122, 2018.

[47] C. Sun, W. He, and J. Hong, "Neural network control of a flexible robotic manipulator using the lumped spring-mass model," IEEE Transactions on Systems, Man, and Cybernetics: Systems, vol. 47, no. 8, pp. 1863 1874,2016

[48] W. He and Y. Dong, "Adaptive fuzzy neural network control for a constrained robot using impedance learning," IEEE Transactions on Neural Networks and Learning Systems, vol. 29, no. 4, pp. 1174-1186, 2018.

[49] C. Yang, C. Chen, N. Wang, Z. Ju, J. Fu, and M. Wang, "Biologically inspired motion modeling and neural control for robot learning from demonstrations," IEEE Transactions on Cognitive and Developmental Systems, vol. 11, no. 2, pp. 281-291, 2018.

[50] S. S. Ge and C. Wang, "Adaptive neural control of uncertain mimo nonlinear systems," IEEE Transactions on Neural Networks, vol. 15, no. 3, pp. 674-692, 2004

[51] C. Yang, X. Wang, Z. Li, Y. Li, and C.-Y. Su, "Teleoperation control based on combination of wave variable and neural networks," IEEE Transactions on Systems, Man, and Cybernetics: Systems, vol. 47, no. 8 , pp. 2125-2136, 2016

[52] Z. Zhu, Y. Xia, and M. Fu, "Attitude stabilization of rigid spacecraft with finite-time convergence," International Journal of Robust and Nonlinear Control, vol. 21, no. 6, pp. 686-702, 2011

[53] S. Yu, X. Yu, B. Shirinzadeh, and Z. Man, "Continuous finite-time control for robotic manipulators with terminal sliding mode," Automatica, vol. 41, no. 11, pp. 1957-1964, 2005.

[54] H.-q. Wang, B. Chen, and C. Lin, "Adaptive neural tracking control for a class of stochastic nonlinear systems," International Journal of Robust and Nonlinear Control, vol. 24, no. 7, pp. 1262-1280, 2014.

[55] Z. Zuo, B. Tian, M. Defoort, and Z. Ding, "Fixed-time consensus tracking for multiagent systems with high-order integrator dynamics," IEEE Transactions on Automatic Control, vol. 63, no. 2, pp. 563-570, 2017.

[56] S. Zhang, Y. Dong, Y. Ouyang, Z. Yin, and K. Peng, "Adaptive neural control for robotic manipulators with output constraints and uncertainties," IEEE Transactions on Neural Networks and Learning Systems, vol. 29, no. 11, pp. 5554-5564, 2018.

[57] S. N. Kumpati, P. Kannan, et al., "Identification and control of dynamical systems using neural networks," IEEE Transactions on neural networks, vol. 1 , no. 1 , pp. 4-27, 1990.

[58] A. U. Levin and K. S. Narendra, "Control of nonlinear dynamical systems using neural networks. ii. observability, identification, and control," IEEE transactions on neural networks, vol. 7, no. 1, pp. 30-42, 1996.

[59] X. Yu, W. He, H. Li, and J. Sun, "Adaptive fuzzy full-state and outputfeedback control for uncertain robots with output constraint," IEEE Transactions on Systems, Man, and Cybernetics: Systems, 2020.

[60] T. H. Lee and C. J. Harris, Adaptive neural network control of robotic manipulators, vol. 19. World Scientific, 1998

[61] W.-H. Zhu and S. E. Salcudean, "Stability guaranteed teleoperation: an adaptive motion/force control approach," IEEE Transactions on Automatic Control, vol. 45, no. 11, pp. 1951-1969, 2000.

[62] P. Malysz and S. Sirouspour, "Nonlinear and filtered force/position mappings in bilateral teleoperation with application to enhanced stiffness discrimination," IEEE Transactions on Robotics, vol. 25, no. 5, pp. 1134-1149, 2009.

[63] S. Sirouspour and P. Setoodeh, "Adaptive nonlinear teleoperation control in multi-master/multi-slave environments," in Proceedings of 2005 IEEE Conference on Control Applications, 2005. CCA 2005., pp. 1263-1268, IEEE, 2005.

[64] H. Zhang, A. Song, H. Li, and S. Shen, "Novel adaptive finite-time control of teleoperation system with time-varying delays and input saturation," IEEE Transactions on Cybernetics, 2019.

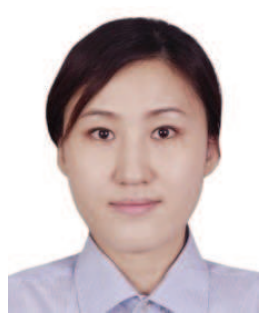

Shuang Zhang (S'11-M'15) received the Ph.D. degree from the Department of Electrical and Computer Engineering, National University of Singapore, Singapore, in 2012. She is currently an Associate Professor with the School of Automation and Electrical Engineering, University of Science and Technology Beijing, Beijing, China. Her current research interests include robotics, adaptive controls, and vibration controls.

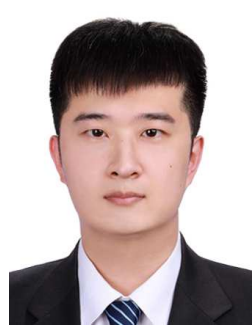

Shuo Yuan received the B.Eng. degree in automation from the School of Automation and Electrical Engineering, University of Science and Technology Beijing, Beijing, China, in 2016, where he is currently pursuing the Master degree. His current research interests include bilateral teleoperation systems, neural network control and adaptive control.

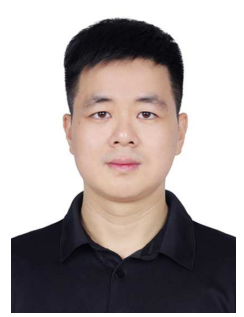

Xinbo Yu (S'16-M'20) received the B.E. degree in control technology and instrument from the School of Automation and Electrical Engineering, University of Science and Technology Beijing, Beijing, China, in 2013 and the Ph.D. degree in control science and engineering from the School of Automation and Electrical Engineering, University of Science and Technology Beijing, Beijing, China, in 2020. $\mathrm{He}$ is currently working as an associate professor in the Institute of Artificial Intelligence, University of Science and Technology Beijing, Beijing, China. His current research interests include adaptive neural networks control, robotics and human-robot interaction.

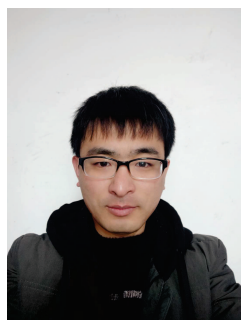

Linghuan Kong received the B.Eng. degree from the College of Engineering, Qufu Normal University, Rizhao, China, in 2016 and the M.Eng. degree from the School of Automation Engineering, University of Electronic Science and Technology of China, Chengdu, China, in 2019. He is currently pursuing the Ph.D. degree with the School of Automation and Electrical Engineering, University of Science and Technology Beijing, Beijing, China. His current research interests include robotics, neural network control, and adaptive control.

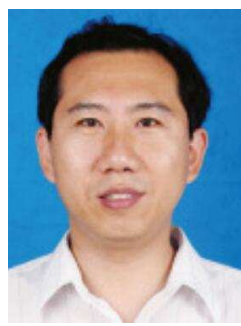

Qing Li received his B.S. degree from North China University of Science and Technology, Tangshan, China, in 1993 and the Ph.D degree in control theory and its applications from University of Science and Technology Beijing, China, in 2000. He is currently a Professor with the School of Automation and Electrical Engineering, University of Science and Technology Beijing, China. His research interests include intelligent control and intelligent optimization. 


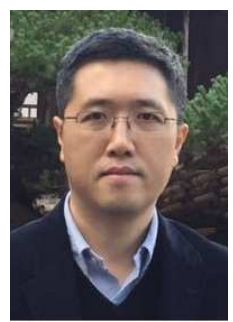

Guang Li (Member, IEEE) received the Ph.D. degree in electrical and electronics engineering, specialized in control systems, from the University of Manchester, Manchester, U.K., in 2007. He is currently a Reader in Control Engineering with the Queen Mary University of London, London, U.K. His current research interests include constrained optimal control, model predictive control, adaptive robust control and control applications, including renewable energies and energy storage. 